\title{
LAMINAR PREMIXED SPRAY FLAME ANALYSIS WITH THERMALLY SENSITIVE INTERMEDIATE KINETICS
}

\author{
J.B. Greenberg* \\ Faculty of Aerospace Engineering, Technion - Israel Institute of Technology, \\ Haifa, Israel \\ and \\ A. Zinoviev, J.W. Dold \\ School of Mathematics, University of Manchester, Manchester M60 1QD, UK
}

* Corresponding author; email: aer9801@ aerodyne.technion.ac.il 


\begin{abstract}
A new thermo-diffusive analysis of one dimensional laminar lean or rich offstoichiometric premixed spray flames has been performed using a chain branching/chain breaking chemical kinetic scheme and under the assumption that the fuel droplets evaporate in a sharp front. The sensitivity of the flame structure, speed and the location of the evaporation front to the initial droplet load have been demonstrated. A linear stability analysis reveals the way in which the spray's presence modifies the neutral stability curves.
\end{abstract}

Keywords: premixed flames, spray flames, stability, chemical mechanism, droplets. 


\section{INTRODUCTION}

The one-dimensional laminar premixed gas flame has served as a cornerstone in the field of combustion, being a relatively simple paradigm for capturing the subtle way in which physico-chemical mechanisms interact under a wide variety of operating circumstances in premixed combustion systems. As spray combustion plays such an important role in many aerospace and other practical applications it is not surprising that premixed laminar spray flames have also been the subject of theoretical/numerical and experimental studies. Experimental studies [1-9] revealed the fact that the very presence of a spray of droplets in the unburned premixture can have a profound impact on the structure, the speed and the stability of the flames they are fueling. An in-depth discussion of the pertinent literature and the somewhat sparse theoretical analyses (see, e.g. [10-12]) can be found in Greenberg et al.[13]. In that work a detailed linear stability analysis of both rich and lean spray flames was carried out. Experimentally observed phenomena [9] of cellular and oscillating spray flames under rich and lean conditions, respectively, were qualitatively reproduced by the theory, and were ascribed to the heat loss mechanism of absorption of heat by the droplets for vaporization together with the usual differential diffusional effects. An extension of [13] to a fuel rich, more realistic polydisperse spray flame was given in [14]. More accurate recent analyses [15-17] concentrated on lean spray flames and demonstrated that, under suitable operating conditions, pulsating instability can be found even if the Lewis number is unity and the evaporation heat loss is neglected. Moreover, for non-unity Lewis number, conditions exist under which there is overlap between the cellular and pulsating instability regions. These new findings were absent from [13] due to certain inherent simplifying assumptions. 
In all these spray flame studies the role of detailed flame chemistry mirrored that of classical gas flame theory inasmuch as a surrogate single global kinetic scheme was adopted to ensure that the correct balance between mass or heat convection and diffusion and exothermic chemical reaction was retained in a satisfactory fashion. However, the success of one-step chemical kinetic models is overshadowed by the fact that the role of intermediate chemical reactants is completely absent, even from some ignition studies (see, e.g. [18]) where chain branching is of utmost importance for determining whether combustion will actually occur or not.

A moderate more realistic extension of the single step chemistry model is based on considering the prime importance of elementary chain branching reactions in controlling the multiple chemical step-ways that make up a full kinetic description. These chain branching steps involve radicals and/or atoms that break down the fuel and/or oxidant thereby producing a radical pool. Ultimately, radicals are also involved in chain breaking/ recombination steps that lead to the appearance of products of reaction.

Zeldovitch et al. [19] were the first to model purely gaseous premixed flames with a chain mechanism using a two-step chemical mechanism. Other work followed using this non-linear mechanism (see, for example, Joulin et al. [20], Chao and Law[21], Mikolaitis[22]) or a sequential mechanism linear in limiting fuel and intermediate concentrations (Margolis and Matkowsky[23]). More recently, a modified Zeldovitch mechanism was suggested by Dold et al.[24-27]. The advantage of the latter mechanism is the fact that it takes the chain breaking or completion step to be linear in the concentration of some intermediate radical or species. This is completely consistent with the final state being an equilibrium one in a broader chemical system and bears the added 
advantage of enabling mathematical tractability (see, in particular, [27] where cogent chemically-based arguments are made for the proposed mechanism).

In the current work we explore a new thermo-diffusive model of a one-dimensional premixed laminar spray flame in which Dold kinetics is taken to describe the chemistry of the flame front in a more general manner than has been hitherto adopted in the spray flame context. Both fuel lean and fuel rich flames are considered. In addition, we investigate the way in which the presence of the spray influences the flame front stability.

\section{GOVERNING EQUATIONS}

Consider a laminar flame front propagating through a fresh homogeneous mixture of fuel vapor, fuel droplets and air. The droplets are viewed from a far-field vantage point, i.e. their average velocity is close to that of their host environment. The droplets in the spray evaporate in a sharp front that is located where the temperature is that of the boiling point of the liquid fuel, $T_{d}$. The temperature of the droplets is taken as equal to that of the surroundings; essentially the droplets heat-up time is small compared to the characteristic time associated with their motion.

It is assumed that the various transport coefficients such as thermal conductivity, diffusion coefficients, specific heat at constant temperature, latent heat of vaporization of the liquid droplets etc. can be satisfactorily specified by representative constant values. Furthermore, the transport properties will be supposed to be determined primarily by the properties of the gaseous species. This follows from the implicit assumption that the liquid fuel volume fraction is sufficiently small, which, for practical purposes, imposes an upper limit on the diameter of droplets in the spray of about $50-100 \mu \mathrm{m}$. 
The modified Zeldovitch-Linan chemical reaction scheme suggested by Dold et al.[2427] is adopted and is formulated here in a general fashion so as to cover both fuel rich and lean off-stoichiometeric premixtures, viz.

$\Re+Z \rightarrow 2 Z \quad(i): \quad k_{B}=A_{B} e^{-T_{B} / T}$

$Z+M \rightarrow P+M \quad$ (ii): $\quad k_{C}=A_{C}$

where $\Re$ denotes the deficient reactant, being the fuel for the fuel lean case and the oxidant for the fuel rich case, $Z$ the intermediate, $P$ the product of reaction and $M$ any molecule that triggers the conversion of the intermediate to the product. Reaction $(i)$ is a high activation energy chain branching reaction having a temperature-sensitive rate coefficient $k_{B}$ with an activation temperature $T_{B}$, whereas reaction $(i i)$ is the chain terminating step (with constant rate coefficient $k_{C}$ ) that is thermally neutral but which provides the dominant exothermal contribution to the heat balance. Typical data for hydrocarbon chemistry readily verifies these assertions (see Dold [27] for a detailed, comprehensive discussion of this matter).

Since the velocity of propagation of the flame is much less than the velocity of sound, dynamic compressibility effects in the mixture can be neglected. Thus, the density becomes a function of the temperature through the gas law. Nevertheless, the ensuing analysis will be carried out in the framework of a thermo-diffusive model for which the effect of gas expansion on the spray flame dynamics is neglected.

The non-dimensional governing equations for the situation at hand take the following form

$$
\begin{aligned}
& L e_{\Re}\left(\partial_{t}+\vec{u} \cdot \nabla\right) \Re=\nabla^{2} \Re-\beta^{2} \mathfrak{R Z k}(T)+n L e_{\Re h} V \\
& L e_{Z}\left(\partial_{t}+\vec{u} \cdot \nabla\right) Z=\nabla^{2} Z+\beta^{2} \mathfrak{R} Z k(T)-Z
\end{aligned}
$$


$\left(\partial_{t}+\vec{u} \cdot \nabla\right) T=\nabla^{2} T+Q Z / L e_{\Re}-L V-a T$

$\left(\partial_{t}+\vec{u}_{d} \cdot \nabla\right) D=-V$

with

$k(T)=\exp (\beta(T-1) /(1+\alpha(T-1))$

Although the general background underlying the form of these equations appears in Dold et al [25] the dimensional governing equations for the two-phase problem and the way in which they are normalized here are explained in the Appendix. Eq.(1) is the conservation equation for the fuel vapor or oxygen mass fraction, $\mathfrak{R}$, depending on whether the premixture is fuel lean or rich, respectively, $L e_{\Re}$ is the appropriate reactant's Lewis number, $\vec{u}$ is the fluid velocity, $\beta$ is the high activation energy of the chain branching reaction, $k(T)$ is the exponential part of the Arrhenius term for the reaction rate of reaction $(i)$ and $V \geq 0$ is the rate of evaporation of fuel droplets. The parameter $n$ is equal to zero for the fuel rich situation and unity for the fuel lean case. Eq(2) describes the conservation of the intermediate species mass fraction $Z$ with $L e_{Z}$ being the relevant Lewis number. Note the third term on the RHS of this equation represents the removal of the intermediate via reaction (ii). Eq.(3) is the energy conservation equation. On the RHS the second term relates to the heat generated by exothermic reaction (ii), the third term to the heat loss incurred by droplet evaporation ( $L$ is associated with the latent heat of vaporization of the liquid fuel) and the fourth term to linear heat loss, with constant $a>0$. Eq.(4) describes the conservation of liquid fuel in the spray of droplets.

The boundary conditions for a flame traveling from right to left are

$\lim _{x \rightarrow-\infty}(\Re, Z, T, D)=(1-n \delta, 0,0, \delta)$ 
$\lim _{x \rightarrow \infty}(\Re, Z, T, D)=\left(0,0, T_{b}, 0\right)$

Here $\delta$ is called the initial droplet load. It represents the ratio of the mass fraction of liquid fuel to that of the total fuel (i.e. fuel liquid + vapor). We shall take the total fuel mass fraction to be constant so that different values of $\delta$ correspond to different combinations of the initial liquid and vapor mass fractions. The temperature $T_{b}$ will be equal to zero in the non-adiabatic case, $a>0$. If $a=0$ it is not hard to show that $T_{b}=Q-L \delta$.

\section{REDUCED BRANCHING REACTION SHEET MODEL}

Suppose the branching reaction (i) occurs at a reaction sheet, $x=b(y, z, t)$, so that the relevant reaction terms are negligible at points off the sheet. In addition, assume that the liquid droplets evaporate infinitely quickly in a front ahead of the reaction front at $x=d(y, z, t)$ where the temperature is $T_{d}$. The governing equations then assume the following form

$$
\begin{aligned}
& L e_{\Re}\left(\partial_{t}+\vec{u} \cdot \nabla\right) \Re=\nabla^{2} \Re \\
& L e_{Z}\left(\partial_{t}+\vec{u} \cdot \nabla\right) Z=\nabla^{2} Z-Z \\
& \left(\partial_{t}+\vec{u} \cdot \nabla\right) T=\nabla^{2} T+Q Z / L e_{F}-a T \\
& \left(\partial_{t}+\vec{u}_{d} \cdot \nabla\right) D=0
\end{aligned}
$$

at points for which $x \neq b(y, z, t)$ and $x \neq d(y, z, t)$. The matching conditions across the evaporation front are readily obtained using continuity of the gaseous species and temperature and by integrating Eqs.(1)-(4) across the front 
$\left[\Re_{n}\right]=-S_{d} L e_{\Re} n \delta, \quad\left[T_{n}\right]=S_{d} L \delta, \quad[D]=-\delta$,

$[\Re]=[Z]=\left[Z_{n}\right]=0, \quad T^{ \pm}=T_{d}$

where the square brackets denote the difference between the enclosed parameters values at $d^{ \pm}$. The subscript $n$ indicates the derivative in the direction normal to the evaporation front and $S_{d}$ is the normal propagation speed of the evaporation front. Across the flame front the conditions are [27]:

$\left[\Re_{n}\right]+\left[Z_{n}\right]=\left[T_{n}\right]=[\Re]=[Z]=0, \quad T^{ \pm}=1$

where $n$ is now the direction normal to the flame front.

\section{PLANAR PREMIXED SPRAY FLAMES}

Suppose the branching reaction sheet is located at $x=0$. Then, a planar premixed spray flame propagating in the negative $x$-direction with speed $S$ will be a steady state solution of the following equations

$$
\begin{aligned}
& L e_{\mathfrak{R}}\left(\Re_{t}+S \Re_{x}\right)=\nabla^{2} \mathfrak{R} \\
& L e_{Z}\left(Z_{t}+S Z_{x}\right)=\nabla^{2} Z-Z \\
& T_{t}+S T_{x}=\nabla^{2} T+Q Z / L e_{\mathfrak{R}}-a T \\
& D_{t}+S D_{x}=0
\end{aligned}
$$

with conditions across the evaporation front

$$
\left[\Re_{x}\right]=-S_{d} L e_{\Re} n \delta, \quad\left[T_{x}\right]=S_{d} L \delta, \quad[D]=-\delta, \quad[\Re]=[Z]=\left[Z_{x}\right]=0, \quad T^{ \pm}=T_{d}
$$

and conditions across the branching reaction sheet $(\beta>>1)$

$$
\left[\mathfrak{R}_{x}\right]+\left[Z_{x}\right]=\left[T_{x}\right]=[\mathfrak{R}]=[Z]=0, \quad T^{ \pm}=1
$$

The boundary conditions as $x \rightarrow \pm \infty$ appear in Equations (6) and (7). 


\section{STEADY STATE SOLUTION}

For the steady state solution depending only on $x$ and having taken $S_{d}=S$ with evaporation at $x=d_{0}$ the solutions of the governing equations can readily be determined.

In the region $-\infty<x \leq d_{0}$

$D=D_{0}(x)=\delta$

$Z=Z_{0}(x)=\frac{L e_{3 i} S}{\gamma_{1}+\gamma_{2}} e^{\gamma_{1} x}$

$\mathfrak{R}=\mathfrak{R}_{0}(x)=1-n \delta-\left(1-n \delta e^{-L e_{\mathfrak{R}} S d_{0}}\right) e^{L e_{F} S x}$

$T=T_{0}(x)=\left(T_{d}+G_{I} e^{\gamma_{1} d_{0}}\right) e^{\mu_{I}\left(x-d_{0}\right)}-G_{I} e^{\gamma_{1} x}$

In the region $d_{0} \leq x \leq 0$

$D=D_{0}(x)=0$

$Z=Z_{0}(x)=\frac{L e_{\Re} S}{\gamma_{1}+\gamma_{2}} e^{\gamma_{1} x}$

$\mathfrak{R}=\mathfrak{R}_{0}(x)=1-e^{\operatorname{Leg}_{\mathfrak{R}} S_{x}}$

$T=T_{0}(x)=\left(1+G_{1}\right) e^{-\mu_{2} x}+H\left(e^{\mu_{1} x}-e^{-\mu_{2} x}\right)-G_{l} e^{\gamma_{1} x}$

and in $0 \leq x<\infty$

$D=D_{0}(x)=0$

$Z=Z_{0}(x)=\frac{L e_{\Re} S}{\gamma_{1}+\gamma_{2}} e^{-\gamma_{2} x}$

$\mathfrak{R}=\mathfrak{R}_{0}(x)=0$

$T=T_{0}(x)=\left(1+G_{2}\right) e^{-\mu_{2} x}-G_{2} e^{-\gamma_{2} x}$

In these solutions 


$$
\begin{aligned}
& \gamma_{1,2}=\frac{1}{2}\left(\sqrt{\left(L e_{Z} S\right)^{2}+4} \pm L e_{Z} S\right), \quad \mu_{1,2}=\frac{1}{2}\left(\sqrt{S^{2}+4 a} \pm S\right) \\
& G_{1}=\frac{Q S}{\left(\gamma_{1}+\gamma_{2}\right)\left(\gamma_{1}-\mu_{1}\right)\left(\gamma_{1}+\mu_{2}\right)}, \quad G_{2}=\frac{Q S}{\left(\gamma_{1}+\gamma_{2}\right)\left(\gamma_{2}-\mu_{2}\right)\left(\gamma_{2}+\mu_{1}\right)} \\
& H\left(e^{\mu_{1} d_{0}}-e^{-\mu_{2} d_{0}}\right)=T_{d}-\left(1+G_{1}\right) e^{-\mu_{2} d_{0}}+G_{l} e^{\gamma_{1} d_{0}}
\end{aligned}
$$

Applying the jump conditions $\left[T_{x}\right]=S L \delta$ at the evaporation front and $\left[T_{x}\right]=0$ at the branching reaction front leads to the following set of two nonlinear algebraic equations to be solved for the evaporation front location and the flame speed, given the values of

$$
\begin{aligned}
& L e_{\mathfrak{R}}, L e_{Z}, Q, \delta, T_{d}, a: \\
& G_{I}\left(\mu_{1} e^{\gamma_{l} d_{0}}+\mu_{2} e^{-\mu_{2} d_{0}}\right)+\mu_{2} e^{-\mu_{2} d_{0}}+\mu_{1} T_{d}=H\left(\mu_{1} e^{\mu_{1} d_{0}}+\mu_{2} e^{-\mu_{2} d_{0}}\right)-S L \delta \\
& G_{I}\left(\gamma_{1}+\mu_{2}\right)+G_{2}\left(\gamma_{2}-\mu_{2}\right)=H\left(\mu_{1}+\mu_{2}\right)
\end{aligned}
$$

It is interesting to note that although the shape of $\mathfrak{R}_{0}(x)$ and the magnitude of $Z_{0}(x)$ depend on $L e_{\mathfrak{R}}$ Eqs (24)-(25) do not. Large activation energy asymptotic analysis using single step global reaction kinetics shows that the flame speed has a Lewis number dependency of roughly $\sqrt{L e_{\mathfrak{R}}}$ [13]. However, the more realistic general description of the chemistry used in the current analysis reveals that the flame speed is independent of $L e_{\mathfrak{R}}$ to the order of approximation considered.

\section{RESULTS - STEADY STATE SOLUTION}

In Fig. 1 we present typical species and temperature profiles for a fuel lean situation for which there is no linear heat loss (i.e. $a=0$ ). For comparative purposes the left hand figure shows the profiles when there is only gaseous fuel initially present in the system. The Lewis number of the intermediate species has been taken as unity - in practice it is 
not likely to be beyond the close proximity of one. Since maximum temperatures rarely exceed $2500 \mathrm{~K}$ taking the unburned temperature to be about $300 \mathrm{~K}$ and the branching temperature to be about 1400K suggest that the realistic values of $Q$ are likely to be in the vicinity of between one and two. The right hand figure corresponds to the case of $\delta=1$. The most striking difference between the two figures lies in the fuel vapor profile. The build up of vapor as the initial liquid fuel load evaporates can be clearly observed. In addition, the altered fuel vapor profile for the spray flame results in a reduction of the maximum value that the intermediate species mass fraction attains at $x=0$. Upon comparing the two temperature profiles the reduction in the maximum temperature due to the presence of the droplets absorbing heat for evaporation is readily seen. (Of course, in the case of a fuel rich spray flame the oxygen and intermediate mass fractions profile will be qualitatively similar to those of the left hand figure (for the fuel and intermediate) whereas the temperature profile alone will be changed as a result of heat lost in evaporating the fuel droplets.) The decrease in the flame temperature is accompanied by a concomitant decrease in the flame speed, and for the fuel lean case with heat loss due to droplet evaporation only the ratio of the spray flame speed to that of an equivalent pure gaseous flame, $S / S_{0}$, is drawn in Fig. 2 for different values of $Q$ and the intermediate species Lewis number. The almost linear decrease of the ratio as the liquid load increases reflects the sort of behaviour predicted using single step kinetics [13]. The drop in the spray flame velocity is sensitive to the value of the heat release parameter $Q$, ranging from a value of about $10 \%$ when $Q=2$ to as much as $100 \%$ when $Q=1.1$ (in this case there is no flame propagation). On the other hand for any given value of $Q$ the intermediate species Lewis number has a minor influence on the flame speed ratio 
causing it to increase slightly for $L e_{Z}>1$ and to decrease for $L e_{Z}<1$, irrespective of the droplet load.

In Fig. 3 flame speeds and the location of the evaporation front are illustrated as functions of the heat loss parameter for different initial droplet loads, for fuel lean flames. The lower branch of each curve would always be associated with dynamically unstable solutions. The case $\delta=0$ corresponds to the single phase gas problem when no droplets are present in the initial unburned mixture. Not unexpectedly, the maximum permitted value of the heat loss parameter for flame propagation decreases as the initial droplet load increases due to the additional heat loss resulting from the evaporation of the droplets. This general behaviour of the flame speed with heat loss concurs with gas flame analyses using a single global chemical reaction [28]. Note, also, that on the upper branch of the solutions the location of the evaporation front moves away from the branching reaction front and the flame speed decreases as the droplet load increases.

In Fig. 4 the influence of the exothermic heat of reaction is illustrated for a droplet load of one. The maximum permitted value of the heat loss parameter grows as $Q$ increases as do the values of the flame speed on the upper branch of the solutions (see left hand part of the figure). The heat loss effect of the liquid fuel exhibits itself inasmuch as the equivalent flame speed curves to those of Fig. 4 but with no initial liquid fuel (i.e. $\delta=0$, not shown here) are displaced to the right. It is interesting to note the way in which the evaporation front location behaves as a function of the heat loss parameter (right hand part of Fig. 4). Sustaining flame propagation clearly goes hand in hand with sustaining an achievable evaporation front. 


\section{LINEAR STABILITY ANALYSIS}

For small amplitude disturbances with a wave-number $k$ and a growth rate $\lambda$ linear stability can be examined by assuming that the branching reaction interface behaves like $x=\varepsilon e^{\lambda t+i k y}$ with $\varepsilon<<1$. The accompanying perturbations in $\mathfrak{R}, Z, T, d$ and $S_{d}$ are taken to have the form

$\left(\mathfrak{R}, Z, T, d, S_{d}\right) \approx\left(\mathfrak{R}_{0}, Z_{0}, T_{0}, d_{0}, S\right)+\varepsilon\left(\mathfrak{R}_{1}(x), Z_{1}(x), T_{1}(x), d_{1},-\lambda d_{1}\right) e^{\lambda t+i k y}$

Substituting in the governing equations (14)-(17) and the matching conditions (18) and (19) and making use of the steady state solutions leads to the following equations

$\mathfrak{R}_{1}^{\prime \prime}-L e_{\mathfrak{R}} S \mathfrak{R}_{1}^{\prime}-\left(L e_{\mathfrak{R}} \lambda+k^{2}\right) \mathfrak{R}_{1}=0$

$Z_{l}^{\prime \prime}-L e_{Z} S Z_{l}^{\prime}-\left(L e_{Z} \lambda+k^{2}+1\right) Z_{l}=0$

$T_{1}^{\prime \prime}-S T_{1}^{\prime}-\left(\lambda+k^{2}+a\right) T=-Q Z_{1} / L e_{\mathfrak{R}}$

$S D_{1}^{\prime}+\lambda D_{1}=0$

in which it can be assumed that $\Re R_{1} \equiv 0$ for $x>0$ and $D_{l} \equiv 0$ for $x>d_{0}$. The appropriate boundary conditions at infinity are

$$
\begin{aligned}
& \lim _{x \rightarrow-\infty}\left(\mathfrak{R}_{1}, Z_{1}, T_{1}, D_{1}\right)=(0,0,0,0) \\
& \lim _{x \rightarrow \infty}\left(Z_{l}, T_{1}\right)=(0,0)
\end{aligned}
$$

and the matching conditions at the steady state evaporation front become

$$
\begin{aligned}
& {\left[\Re_{R_{1}^{\prime}}^{\prime}\right]=L e_{\Re} \delta n d_{1}\left(\lambda+L e_{\mathfrak{R}} S^{2}\right), \quad\left[T_{1}^{\prime}\right]=-L \delta d_{1} \lambda-d_{1}\left[T_{0}^{\prime \prime}\right], \quad\left[Z_{I}\right]=\left[Z_{1}^{\prime}\right]=0} \\
& {\left[\mathfrak{R}_{1}\right]=L e_{\mathfrak{R}} S \delta n d_{1}, \quad\left[T_{1}\right]=-L S \delta d_{1}, \quad T_{1}^{-}=-d_{l}\left(\mu_{1} T_{d}+e^{\gamma_{1} d_{0}}\left(\mu_{l}-\gamma_{l}\right) G_{I}\right)}
\end{aligned}
$$

whereas those at the reaction front are 


$$
\begin{aligned}
& {\left[\mathfrak{R}_{1}^{\prime}\right]+\left[Z_{1}^{\prime}\right]=\left(L e_{Z}-L e_{\mathfrak{R}}\right) L e_{\mathfrak{R}_{R}} S^{2}, \quad\left[T_{1}^{\prime}\right]=0} \\
& {\left[\mathfrak{R}_{1}\right]=-L e_{\mathfrak{R}_{R}} S, \quad\left[Z_{1}\right]=L e_{\mathfrak{R}} S, \quad T_{1}=\mu_{2}-G_{2}\left(\gamma_{2}-\mu_{2}\right)}
\end{aligned}
$$

The solution of these equations subject to the boundary and matching conditions are as follows.

In the region $-\infty<x \leq d_{0}$

$$
D_{1}(x)=0
$$

$Z_{1}(x)=P e^{\gamma_{3} x}$

$\mathfrak{R}_{1}(x)=\left(L e_{\mathfrak{R}} S+R\left(e^{-v_{l} d_{0}}-e^{v_{2} d_{0}}\right)-L e_{\mathfrak{R}} S \delta n d_{1} e^{-v_{l} d_{0}}\right) e^{v_{l} x}$

$T_{1}(x)=M_{l} e^{\mu_{3} x}+K_{l} e^{\gamma_{3} x}$

In the region $d_{0} \leq x \leq 0$

$$
D_{1}(x)=0
$$

$Z_{1}(x)=P e^{\gamma_{3} x}$

$\mathfrak{R}_{l}(x)=L e_{\mathfrak{R}} S e^{v_{I} x}+\operatorname{Re}^{v_{2} d_{0}}\left(e^{-v_{2} x}-e^{v_{I} x}\right)$

$T_{1}(x)=\left(\mu_{2}-G_{2}\left(\gamma_{2}-\mu_{2}\right)-K_{1}\right) e^{\mu_{3} x}+M_{2}\left(e^{-\mu_{4} x}-e^{\mu_{3} x}\right)+K_{1} e^{\gamma_{3} x}$

and in $0 \leq x<\infty$

$D_{1}(x)=0$

$Z_{l}(x)=\left(P+L e_{\mathfrak{R}} S\right) e^{-\gamma_{4} x}$

$\mathfrak{R}_{1}(x)=0$

$T_{1}(x)=\left(\mu_{2}-G_{2}\left(\gamma_{2}-\mu_{2}\right)-K_{2}\right) e^{-\mu_{4} x}+K_{2} e^{-\gamma_{4} x}$

where 


$$
\begin{aligned}
& v_{1,2}=\frac{1}{2}\left(\sqrt{4\left(L e_{\mathfrak{R}} \lambda+k^{2}\right)+L e_{\mathfrak{R}}^{2} S^{2}} \pm L e_{\mathfrak{R}} S\right) \\
& \gamma_{3,4}=\frac{1}{2}\left(\sqrt{4\left(L e_{Z} \lambda+k^{2}+1\right)+L e_{Z}^{2} S^{2}} \pm L e_{Z} S\right) \\
& \mu_{3,4}=\frac{1}{2}\left(\sqrt{4\left(\lambda+k^{2}+a\right)+S^{2}} \pm S\right) \\
& R=\frac{1}{v_{1}+v_{2}}\left(v_{l} S-\lambda-L e_{\mathfrak{R}_{R}} S^{2}\right) L e_{\mathfrak{R}_{R}} d_{l} \delta n \\
& P=\frac{1}{\gamma_{3}+\gamma_{4}}\left(R\left(v_{1}+v_{2}\right) e^{v_{2} d_{0}}-L e_{\mathfrak{R}} S\left(v_{1}+\gamma_{4}\right)+\left(L e_{\mathfrak{R}}-L e_{Z}\right) L e_{\mathfrak{R}} S^{2}\right) \\
& K_{1}=\frac{P Q}{L e_{\mathfrak{R}}\left(\mu_{3}-\gamma_{3}\right)\left(\mu_{4}+\gamma_{3}\right)}, \quad K_{2}=\frac{Q\left(L e_{\mathfrak{R}} S+P\right)}{L e_{\mathfrak{R}}\left(\mu_{4}-\gamma_{4}\right)\left(\mu_{3}+\gamma_{4}\right)} \\
& M_{1}=-K_{l} e^{d_{0}\left(\gamma_{3}-\mu_{3}\right)}-d_{l} e^{-\mu_{3} d_{0}}\left(\mu_{1} T_{d}+G_{I} e^{\gamma_{1} d_{0}}\left(\mu_{1}-\gamma_{1}\right)\right) \\
& M_{2}\left(e^{-\mu_{4} d_{0}}-e^{\mu_{3} d_{0}}\right)=-S L \delta d_{1}+\left(K_{1}+M_{1}+G_{2}\left(\gamma_{2}-\mu_{2}\right)-\mu_{2}\right) e^{\mu_{3} d_{0}}
\end{aligned}
$$

The unexploited conditions $\left[T_{1}^{\prime}\right]=0$ at $x=0^{ \pm}$and $\left[T_{1}^{\prime}\right]=-L \delta d_{1} \lambda-d_{1}\left[T_{0}^{\prime \prime}\right]$ at $x=d_{0}^{ \pm}$ (with $\left[T_{0}^{\prime \prime}\right]$ derivable from Eqs (20d) and (21d)) lead to the following dispersion relationships which define the growth rate $\lambda$ and $d_{1}$ as functions of the wave-number $k$ and all other parameters of the system $\left(Q, S, L e_{\mathfrak{R}}, L e_{Z}, L, \delta, T_{d}\right.$ and $\left.a\right)$ :

$$
\begin{aligned}
& \frac{P Q}{L e_{\mathfrak{R}}}\left(\frac{1}{\mu_{4}+\gamma_{3}}+\frac{1}{\mu_{3}+\gamma_{4}}\right)+\frac{Q S}{\mu_{3}+\gamma_{4}}+\left(\mu_{3}+\mu_{4}\right)\left(\frac{Q S}{\left(\gamma_{1}+\gamma_{2}\right)\left(\gamma_{2}+\mu_{1}\right)}+M_{2}-\mu_{2}\right)=0 \\
& L \delta d_{1}\left(\lambda+S\left(\mu_{1}-\mu_{3}\right)\right)-M_{2}\left(\mu_{3}+\mu_{4}\right) e^{-\mu_{4} d_{0}}+e^{-\mu_{2} d_{0}} \mu_{2}\left(\mu_{1}+\mu_{2}\right) d_{1}\left(G_{1}-H+1\right)=0
\end{aligned}
$$


Given a steady state solution that satisfies Eqs. (24) and (25) the dispersion relations can be solved numerically to determine the linear stability characteristics of the premixed spray flame.

\section{RESULTS - STABILITY ANALYSIS}

Armed with a steady state solution the dispersion relations (39a) and (39b) can be solved to find the dependence of the least stable growth rate $\lambda$ on the wave-number $k$. Henceforth, only adiabatic flames, $a=0$, will be considered

Fuel lean spray flames

In Fig. 5 we show typical results for fuel lean spray flames for which $L e_{F}<1$ and for various initial droplet loads. (Note that the symbol $\mathfrak{R}$ becomes $F$ for the fuel lean case). For $L e_{F}<1$ the least stable root appears to be real. It is always zero for $k=0$ but elsewhere it varies with $k$. For non-zero wave numbers it first becomes positive as $L e_{F}$ decreases through a threshold value. Neutral stability curves are drawn in Fig. 5. For conditions above any given curve the flame will be stable whereas for conditions below the curve the flame will develop cellular instability. It is interesting to observe that for this lean fuel case the presence of the spray appears to enhance stability. This is qualitatively similar to what was found in [13] for a finite rate of evaporation, using a near equi-diffusion flame analysis with a single step global chemical model. However, the effect of the spray is far more pronounced in the current work as well as not being monotonically decreasing for all droplet loads. Indeed, for $\delta \geq 0.5$ the maximum value of the wave-number is found to be non-zero thus departing from the purely gaseous flame classical behaviour.

In Fig. 6 the neutral stability curves are plotted for a higher value of the non-dimensional latent heat of vaporization, $L$. On comparison of Figs. 5 and 6 it is evident that the 
sensitivity of the curves to the value of $L$ is rather dramatic. It can be seen in Figure 6 that for $\delta=0.65$ the curve transcends that of the purely gaseous flame and a small region exists in which a cellular flame will be observed even though an equivalent gaseous flame will be stable. An insight into this seemingly anomalous behaviour is afforded by Fig. 7 which is based on the expression for the flame temperature, $T_{b}=Q-L \delta$. From Eq.(13) it is known that the normalized temperature at $x=0$ is unity. Therefore, given the heat of reaction and the latent heat of vaporization the limiting droplet load at which $T_{b}$ drops to unity is given by $\delta=(Q-1) / L$. Now take $Q=1.2$ and $L=0.3$. This implies that the limiting value of $\delta$ is $2 / 3$ which is close to the value of the liquid droplet load for which the anomalous behaviour of Fig. 6 is seen to occur. Therefore, use of Fig. 7 can help to predict under which circumstances limiting behaviour will result. In practical terms a stable flame will probably cease to exist under such circumstances. This is borne out in Fig. 8 in which a curve corresponding to the limiting curve of Fig. 6 is shown. The reciprocal of the evaporation front location is plotted as a function of the liquid droplet load. It can be seen that $-d_{0}^{-1}$ tends to zero as $\delta$ tends to $2 / 3$. This corresponds to a case of flame extinction, or $d_{0} \rightarrow-\infty$. A different view of Fig. 6 is presented in Fig. 9 and gives an inkling of the sort of scaling that is involved. Two sets of curves are shown: (1) neutral stability type curves for values of $\delta$ ranging from 0.4 to 0.65 in steps of 0.01 (as $\delta$ increases the curves of $L e_{F}$ shift to the right) and (2) $d_{1}$ (which is associated with the perturbation to the steady state vaporization front location) for the same range of values of $\delta$, both plotted against $-d_{0} k$. The first curves show that the stability behaviour is intimately tied in with wavelengths of the order of $d_{0}$. The 
curves for $d_{1}$ are almost completely insensitive to the droplet load and tend to zero as $-d_{0} k$ grows.

The results discussed so far have related to fuel Lewis numbers less than unity. But for many realistic fuels this is not the case and $L e_{F}>1$. For such values the existence of oscillatory instabilities is known from high activation energy asymptotics of gas flames with a single global chemical kinetic scheme. In Fig. 10 neutral stability curves are drawn for oscillatory instability for various droplet loads. The region above a given curve is that of oscillatory instability whereas that below it is of stable behaviour. It is evident that the presence of the droplets in the system can facilitate the onset of oscillatory instability (this was observed experimentally by Atzler[9]) even for $L e_{F} \leq 1$. Suard et al. [15] appear to be the first to have demonstrated numerically that a pulsating mode of propagation of laminar premixed spray flames can occur when the vaporization time is smaller than the reaction time (a single chemical reaction was assumed) even for a unity Lewis number. Nicoli et al [17] performed a mathematical analysis using a single step chemical model and found that for $L e_{F} \leq 1$ oscillatory instability can occur. The current model establishes that this is also possible using a more general chemical kinetic scheme and is inherent to this two phase situation. The neutral stability boundaries for both oscillatory and cellular instabilities are assimilated into single graphs in Fig.11, for three values of the droplet load. It is remarkable that as the droplet load increases the two curves grow closer together. For $\delta$ less than or equal to about 0.5 conditions exist for which stable spray flame propagation is possible over a narrow range of fuel Lewis number. However, as $\delta$ further increases the onset of instability of one sort or another will always occur due to the overlay of both regions of instability. It is interesting to note that this enhancement of 
the oscillating instability bears some similarity to what is found in analysis of condensed two phase flame propagation [29].

The way in which the overlap of regions of cellular and oscillatory instability is influenced by $Q$ is shown in Fig. 12. It is readily seen that as the heat of the recombination reaction (ii) increases the domain of stable operating conditions grows. This coincides with the findings of Dold et al. [25] for purely gas flames.

In Fig. 13 the sensitivity of the neutral cellular and oscillatory stability curves to the value of $L e_{Z}$ is examined. The previously discussed minor role of the intermediate species' Lewis number (see Fig. 2 and accompanying remarks) reappears in the stability analysis. For realistic values of the fuel Lewis number the intermediate species has little influence on the region of stable operating conditions.

\section{Fuel rich spray flames}

Turning to fuel rich spray flames, neutral stability curves are drawn in Fig. 14. (Recall that the symbol $\mathfrak{R}$ becomes $O$ for the fuel rich case.) Once again the region above a given curve is a region of stable operating conditions whereas below it is unstable. In stark contrast to the equivalent fuel lean case (Fig. 5) it is seen that the presence of the droplets in the system tends to promote cellular instability for small values of the wave number. This is in consonance with the behaviour predicted in [13] using a single chemical reaction model and experimental evidence [9]. For a higher value of the latent heat of vaporization the neutral stability curves of Fig. 15 are predicted. The region of spray flame instability is now extended beyond $L e_{O}=1$ (compare with Fig. 14). However, as was explained for the fuel lean case, the neutral stability curves do not exist for $\delta \geq 2 / 3$. 
Neutral oscillatory stability curves are shown in Fig. 16. The penetration of the curves into practically realizable regions in the vicinity of $L e_{O}=1$ is clearly visible. In fact, a more complete picture illustrating both oscillatory and cellular regions of instability as a function of the droplet load can be seen in Fig. 17 for three values of the intermediate species' Lewis number. There is much similarity between this figure and the equivalent figure for the fuel lean spray flames (Fig. 13) (including the relative insensitivity of the curves to the value of $L e_{Z}$ ) although for practical values of $L e_{O}$ in the proximity of unity cellular instability is more likely to arise than oscillatory instability. This enhanced propensity for the formation of cellular instability (rather than oscillatory instability) in fuel rich spray flames was observed by Atzler[9] and it was suggested [13] that the heat loss due to droplet evaporation was responsible for it. In order to examine this hypothesis the endothermic spray related term can be effectively removed by setting $L=0$. Mathematically this corresponds to a purely gaseous fuel-rich flame, even when there are droplets, because they then have no influence on the problem through either oxidant concentration or the temperature. In Fig. 18 the way in which the critical Lewis numbers at the boundaries of cellular and oscillatory stability change as a function of $Q$ is drawn for $L=0$ and $L=0.3$. The upper/lower curves delineate the onset of oscillatory/cellular oscillations. Within the wedge-shaped regions in between the upper and lower curves operating conditions ensure stable spray flames. However, it is interesting to note that for the case of no droplet heat loss (which would be somewhat artificial when there actually are droplets) a practically plausible region of cellular instability with an oxidant Lewis number that is below one, or an oscillatory instability for Lewis number greater than one, is attainable if the heat of reaction $Q$ is close enough to unity. Thus, it is concluded that 
although droplet heat loss does play a role in the onset of instabilities in premixed spray flames the primary cause of the onset is of a more complex nature and may be along the lines suggested by Nicoli et al. [16,17] whereby the perturbed movement of the point where evaporation occurs becomes responsible for a feed-back mechanism between itself and heat and mass transfer to the flame front. Nevertheless, for the larger value of $L=0.3$ (and larger more realistic values of $Q$ ) Fig. 18 does imply that the droplets heat loss is responsible for a greater sensitivity to cellular instability.

\section{CONCLUSIONS}

A new analysis of one dimensional laminar lean or rich premixed spray flames has been performed using a chain branching/chain breaking chemical kinetic scheme and under the assumption that the fuel droplets evaporate in a sharp front. The flame structure, speed and location of the evaporation front were shown to be sensitive to the droplet load. The flame speed was found to be insensitive to the Lewis number of the intermediate species. A linear stability analysis was also carried out for both fuel lean and fuel rich spray flames. It was found that both cellular and oscillatory instabilities are possible for practical ranges of the relevant gas and spray related parameters. For operating conditions under which there is no overlap of oscillating and cellular instability regions the presence of the droplets can enhance/inhibit cellular instability depending on whether the flame is fuel rich or fuel lean. It was also demonstrated that the major cause of spray flame instabilities is not heat loss due to the evaporating droplets as was previously suggested. Rather a feed-back mechanism resulting from perturbed motion of the evaporation front, as advocated in the literature, may be at the root of the instabilities. Finally, it is encouraging that the predictions of the current model, involving a more general chemical 
kinetic mechanism than has been hitherto considered in the spray combustion theory context, are in qualitative agreement with predictions of laminar spray flame models that used a single chemical kinetic mechanism. Moreover, because of the structure of the equations implied by the chain branching/chain breaking mechanism the present model enables a wider scope of operating conditions to be considered and greater insight to be achieved into this difficult two-phase problem than was previously possible.

\section{ACKNOWLEDGEMENTS}

J.B.G. acknowledges the partial support of the Technion Fund for Promotion of Research and a grant from the US-Israel Bi-National Science Foundation (2004069). Thanks are also due to the Manchester Institute for Mathematical Sciences at Manchester University, UK where part of this work was carried out during a sabbatical leave.

\section{APPENDIX}

In this appendix we present the governing equations in dimensional form and then explain how they are recast into the compact non-dimensional form (1)-(4). Most of the rescaling and definitions follow [25] but there are notable differences that arise due to the fact that a two-phase problem is being solved here.

The governing equations conservation equations are

$$
\begin{aligned}
& \rho\left(\partial_{t^{*}}+\vec{u}^{*} \cdot \nabla^{*}\right) Y_{\Re R}=\rho D_{9 R} \nabla^{* 2} Y_{\Re R}-W_{P R} \omega_{B}+n V^{*} \\
& \rho\left(\partial_{t^{*}}+\vec{u}^{*} \cdot \nabla^{*}\right) Y_{Z}=\rho D_{Z} \nabla^{* 2} Y_{Z}+W_{Z} \omega_{B}-W_{Z} \omega_{C} \\
& \rho C_{p}\left(\partial_{t^{*}}+\vec{u}^{*} \cdot \nabla^{*}\right) T^{*}=\lambda \nabla^{* 2} T^{*}+Q^{*} \omega_{C}-l^{*}-L^{*} V^{*} \\
& \rho\left(\partial_{t^{*}}+\vec{u}_{d}^{*} \cdot \nabla^{*}\right) Y_{d}=-\rho V^{*}
\end{aligned}
$$

where 
$\omega_{B}=A_{B} \frac{\rho Y_{\mathfrak{R}}}{W_{\mathfrak{R}}} \frac{\rho Y_{Z}}{W_{Z}} e^{-T_{B} / T^{*}}, \quad \omega_{C}=A_{C} \frac{\rho Y_{Z}}{W_{Z}} \frac{\rho}{W}, \quad V^{*}=C^{*} Y_{d} \hat{H}\left(T^{*}-T_{d}^{*}\right)$

In these equations $\rho$ is the density, $Y_{i}$ is the mass fraction of the deficient reactant, the intermediate and the liquid fuel in the droplets for $i=\Re, Z$ and $d$, respectively, $D_{i}$ is the diffusion coefficient and $W_{i}$ is the molecular weight of the deficient reactant and the intermediate for $i=\mathfrak{R}, Z$, respectively, $W$ is the mean molecular weight, $C_{p}$ is the specific heat of the mixture and $\lambda$ is its thermal conductivity, $Q^{*}$ is the energy released in converting a mole of the intermediate species into products, $l^{*}$ is the rate of heat loss to infinity by radiation and $\vec{u}$ and $\vec{u}_{d}$ are the velocities of the gas and liquid phases, respectively, and $L^{*}$ is the latent heat of evaporation. In the reaction rates, $\omega_{B}$ and $\omega_{C}$, of the branching and completion steps $A_{B}, A_{C}$ are the pre-exponential coefficients and $T_{B}$ is the activation temperature. The rate of evaporation is given by the expression for $V^{*}$ derived from the sectional approach to spray modeling, with $C^{*}$ being the appropriate evaporation coefficient when the rate of evaporation is finite. $\hat{H}$ is the Heaviside function and its argument indicates that the onset of significant evaporation occurs when the temperature is that of the liquid fuel's boiling temperature, $T_{d}^{*}$. In Eq.(A1) for the deficient reactant, $\mathfrak{R}$, the parameter $n$ assumes the value zero or one depending on whether the pre-mixture is fuel rich or lean, respectively.

Following [25] the aforementioned equations are transformed to dimensionless form using the rescaling

$t^{*}=t_{s} t, \quad \underline{x}^{*}=r_{s} \underline{x}, \quad T^{*}=T_{0}^{*}+\left(T_{b}-T_{0}^{*}\right) \tilde{T}, \quad Y_{\mathfrak{R}}=Y_{\mathfrak{R}} \mathfrak{R}, \quad Y_{Z}=Y_{Z s} Z, \quad Y_{d}=Y_{F 0} D$

with 


$$
\begin{aligned}
& r_{s}^{2}=\frac{D_{Z} W}{\rho A_{C}}, \quad t_{s}=\frac{\rho C_{p}}{\lambda} r_{s}^{2}, \quad\left(\vec{u}^{*}, \vec{u}_{d}^{*}\right)=\frac{r_{s}}{t_{s}}\left(\vec{u}, \vec{u}_{d}\right), \quad l=\frac{\rho C_{p} T_{b}}{t_{s}}\left(1-\tilde{T}_{0}\right) l^{*}, \\
& Q=\frac{C_{p} T_{b} W_{\Re R}}{Y_{\mathfrak{R} 0}}\left(1-\tilde{T}_{0}\right) Q^{*}, \quad \theta^{2} e^{\theta}=\frac{W A_{B}}{W_{F} A_{C}} Y_{\mathfrak{R}}, \quad T_{b}=\frac{T_{B}}{\theta}, \\
& L e_{\mathfrak{R}}=\frac{\lambda}{\rho C_{p} D_{\mathfrak{R}}}, \quad L e_{Z}=\frac{\lambda}{\rho C_{p} D_{Z}}, \quad Y_{Z s}=\frac{L e_{Z} W_{Z}}{L e_{F} W_{\mathfrak{R}}} Y_{\mathfrak{R} 0}, \quad T_{0}^{*}=T_{b} \tilde{T}_{0}, \\
& Y_{\mathfrak{R}}=\left(n Y_{F 0, \text { total }}+(1-n) Y_{O 0}\right) \Re, \quad Y_{d}=Y_{F 0, \text { total }} D, \quad Y_{F 0, \text { total }}=Y_{F 0}+Y_{d 0}, \\
& L=\frac{L^{*} Y_{F 0, \text { total }}}{C_{p} T_{b}\left(1-\tilde{T}_{0}\right)}, \quad C=\frac{\tilde{C}}{t_{s}}, \quad T=\frac{\tilde{T}-\tilde{T}_{0}}{1-\tilde{T}_{0}}
\end{aligned}
$$

In these definitions $Y_{F 0, \text { total }}$ is the total mass fraction of fuel (i.e. vapor + liquid) in the fresh mixture. Making use of the above in Eqs.(A1)-(A5) leads to the non-dimensional form of the governing equations Eqs.(1)-(5) which are treated in the text. 


\section{REFERENCES}

1. Briffa, E.F. \& Dombrowski, N. 1970 Proc. Roy. Soc. Lond. A 326, 309-322.

2. Mizutani, Y. \& Nakajima, A. 1973a Combust. Flame 20, 343-350.

3. Mizutani, Y. \& Nakajima, A. 1973b Combust. Flame 20, 351-357.

4. Polymeropoulos, C.E. 1974 Combust. Sci. Technol. 9, 197-207.

5. Hayashi, S., Kumagai, S. 1974 Fifteenth Symposium (International) on Combustion, The Combustion Institute, Pittsburgh, Penn., 445-452.

6. Polymeropoulos, C.E \& Das, S. 1975 Combust. Flame 25, 247-257.

7. Hayashi, S., Kumagai, S. \& Sakai, T. 1976 Combust. Sci. Technol. 15, 169-177.

8. Ballal, D.R. \& Lefebvre, A.H. 1981 Eighteenth Symposium (International) on Combustion, The Combustion Institute, Pittsburgh, Penn., 321-328.

9. Atzler, F. 1999 Fundamental Studies of Aerosol Combustion, Ph.D. Thesis, School of Mechanical Engineering University of Leeds.

10. Lin, T.H., Law, C.K. \& Chung, S.H. 1988 Int. J. Heat Mass Transfer 31, 1023-1034.

11. Lin, T.H. \& Sheu, Y.Y. 1991 Combust. Flame 84, 333-342.

12. Silverman, I., Greenberg, J.B. \& Tambour, Y. 1991, SIAM J. Appl. Math. 51, 12841303.

13. Greenberg, J.B., A.C. McIntosh and J. Brindley, 2001, Proc. Roy. Soc. Lond. A, 357, $1-31$.

14. Greenberg, J.B., Atom. Sprays, 2002, 12, 123-143.

15. Suard, S., Haldenwang, P. and Nicoli, C., 2004, C.R. Mecanique, 32, 387-396.

16. Nicoli, C., Haldenwang, P. and Suard, S., 2005, Combust. Flame 143, 299-312.

17. Nicoli, C., Haldenwang, P. and Suard, S., 2007, Combust. Flame 149, 295-313. 
18. Kapila, A.K.,1981, Int. J. Engn. Sci., 19, 495-509.

19. Zeldovitch, Ya. B., Barrenblatt, G.I., Librovich, V.B. and Makhviladze, G.M., The Mathematical Theory of Combustion and Explosions, Consultants Bureau, New York, 1985.

20. Joulin, G., Linan, A., Ludford, G.S.S, Peters, N. and Schmidt-Laine, C., 1985, SIAM J. Appl. Math.. 45, 420-434.

21. Chao, B.H. and Law, C.K., 1994, Int. J. Heat Mass Trans., 37, 673-680.

22. Mikolaitis, D.W., 1986, Combust. Sci. Technol., 49, 277-288.

23. Margolis, S.B. and Matkowsky, B.J., 1982, SIAM J. Appl. Math., 42(6), 11751188.

24. Dold, J.W., Thatcher, R.W.Omon-Arancibia, A. and Redman, J., 2002, Proc. Comb. Inst. , 29, 1519-1526.

25. Dold, J.W., Daou, J. and Weber, R.W., 2004, in "Simplicity, Rigor and Relevance in Fluid Mechanics", F,J, Higuera, J. Jimenaz and J.M. Vega (eds) CIMNE Publishers, Barcelona.

26. Dold, J.W., Weber, R.D., Thatcher, R.W. and Shah, A.A., 2003, Combust. Theory Modeling, 7, 175-203.

27. Dold, J.W., 2007, Combust. Theory Model., 11, 909-948.

28. Buckmaster, J.D. and Ludford, G.S.S, 1982, Theory of Laminar Flames, Cambridge University Press, Cambridge, UK.

29. Margolis, S.B., 1983, SIAM J. Appl. Math., 43(2), 351-369. 

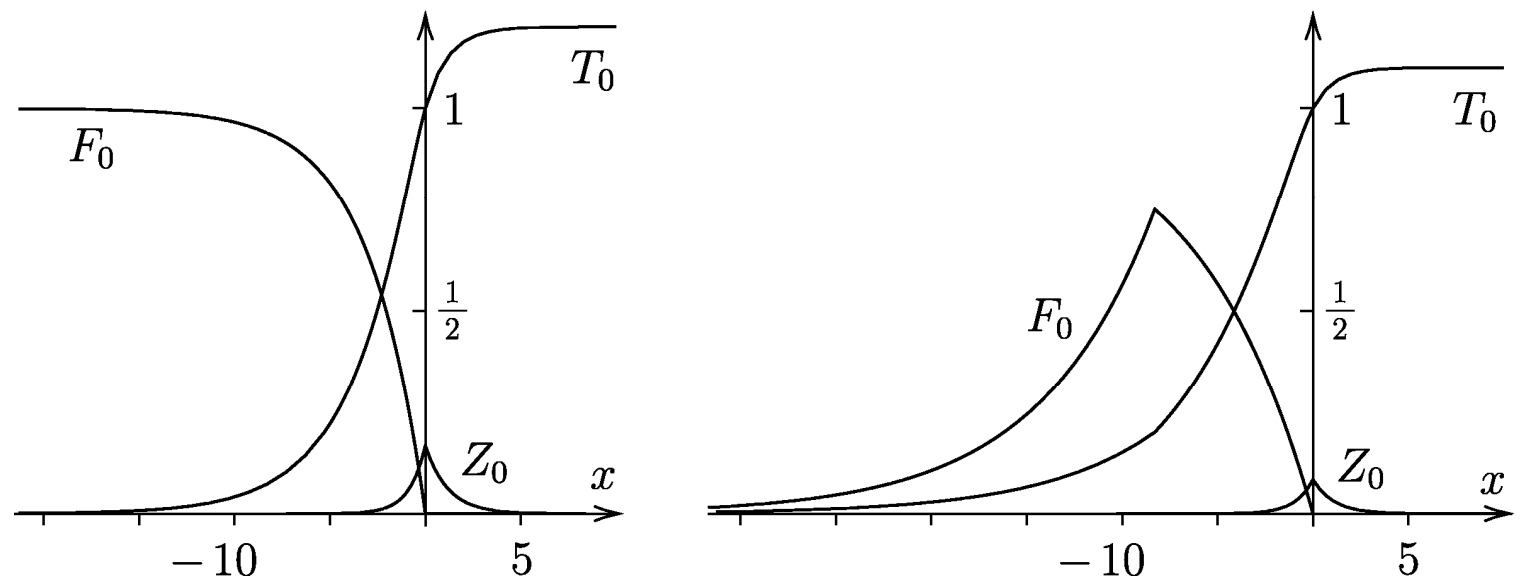

Figure 1: Typical lean reactant, intermediate species and temperature profiles for a purely gaseous flame ( $\delta=0$, left figure) and a spray flame ( $\delta=1$, right figure); $a=0, Q=1.2, L e_{Z}=1, L=0.1, T_{d}=0.2$. 


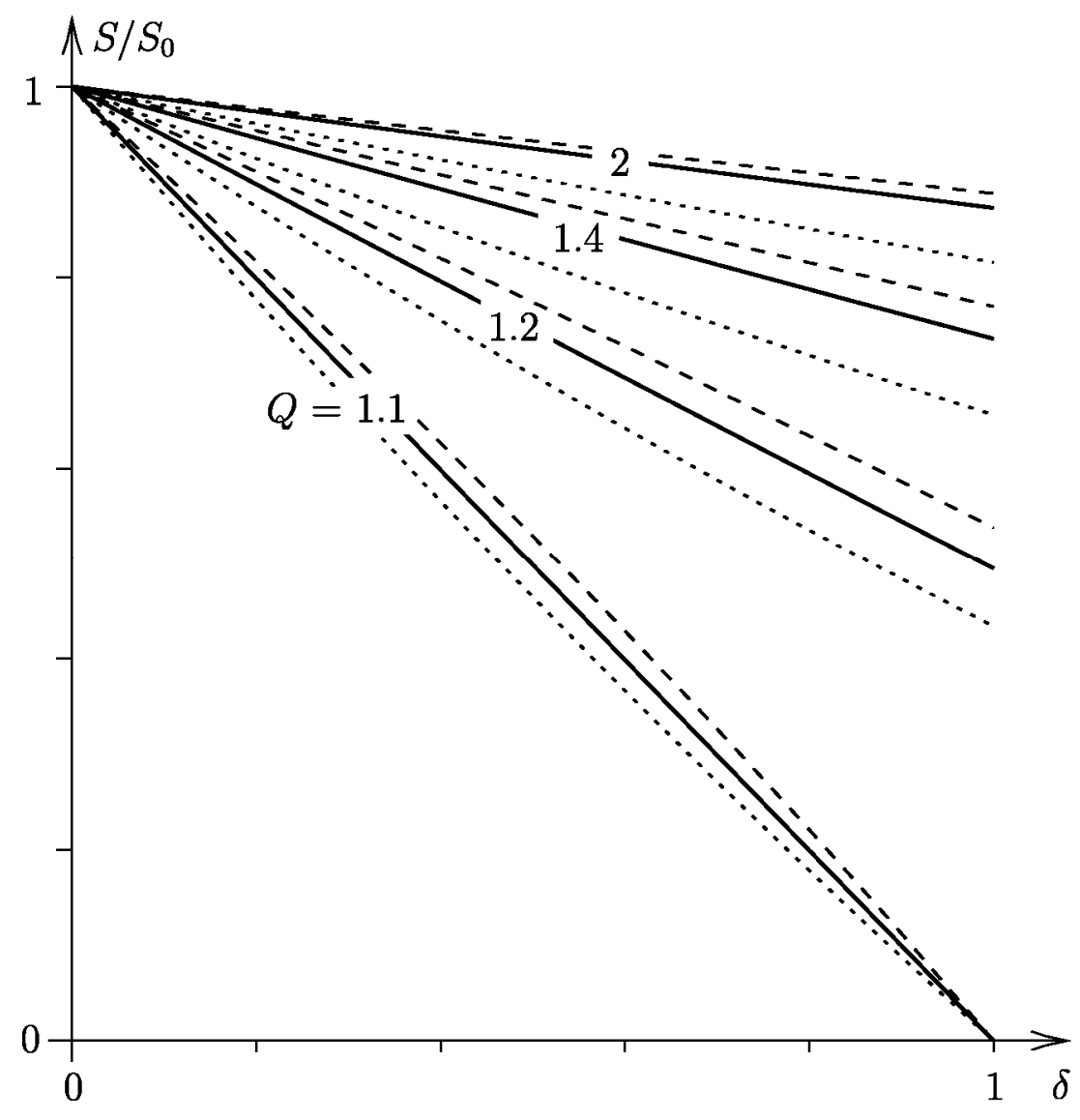

Figure 2: Ratio of fuel lean spray flame speed to equivalent gaseous flame speed, $S / S_{0}$, as a function of initial liquid fuel load, for selected values of $Q$; $a=0, L=0.1, T_{d}=0.2$; Key: Solid lines $-L e_{Z}=1$, Dashed lines $-L e_{Z}=2$, Dotted lines $-L e_{Z}=0.25$. 

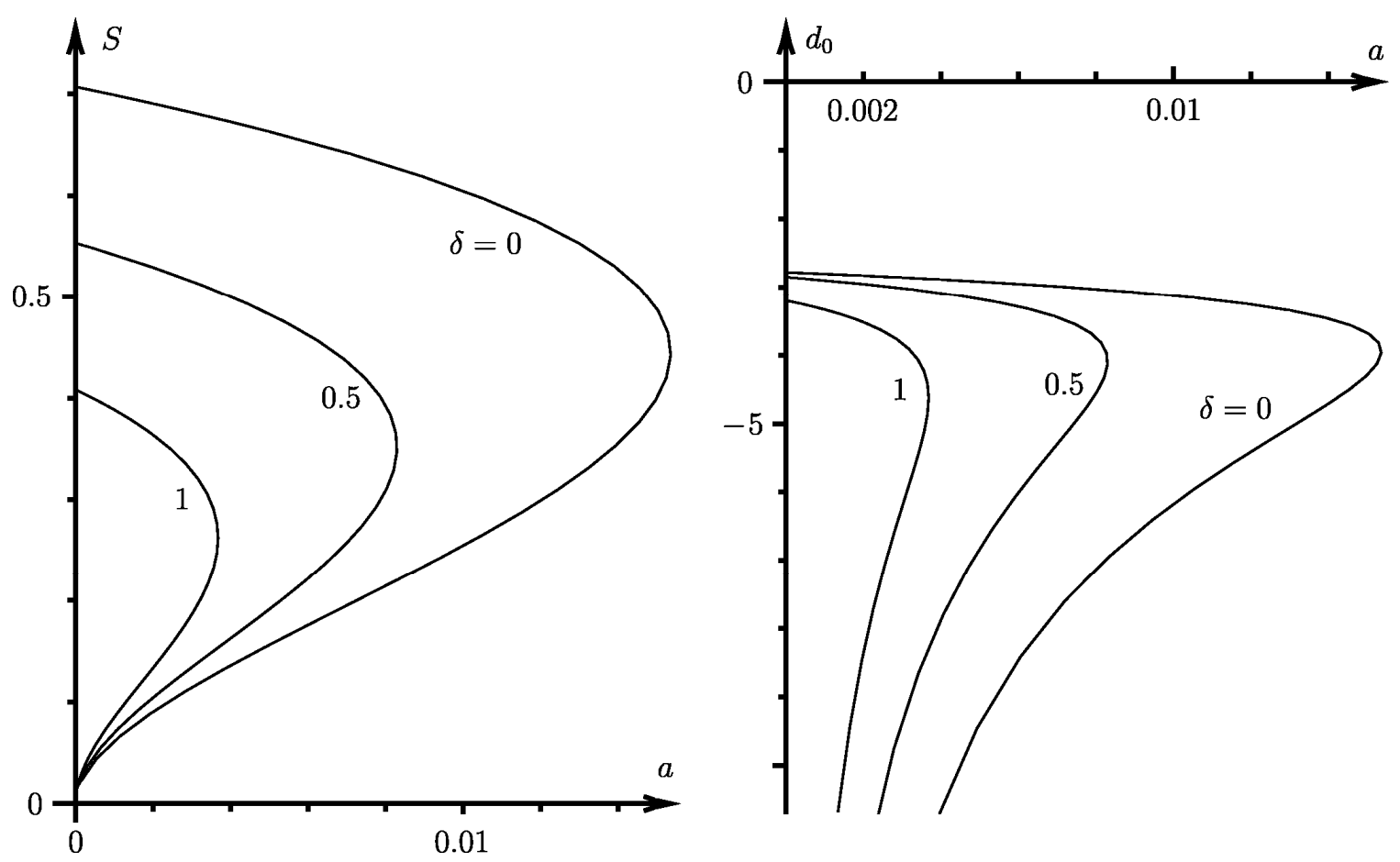

Figure 3: Dependence of the flame speed, $S$ (left figure) and the evaporation front location, $d_{0}$ (right figure) on the heat loss parameter $a$ for selected values of the initial droplet load; $L e_{Z}=1, L=0.2, T_{d}=0.2, Q=1.5$, fuel lean flame. 

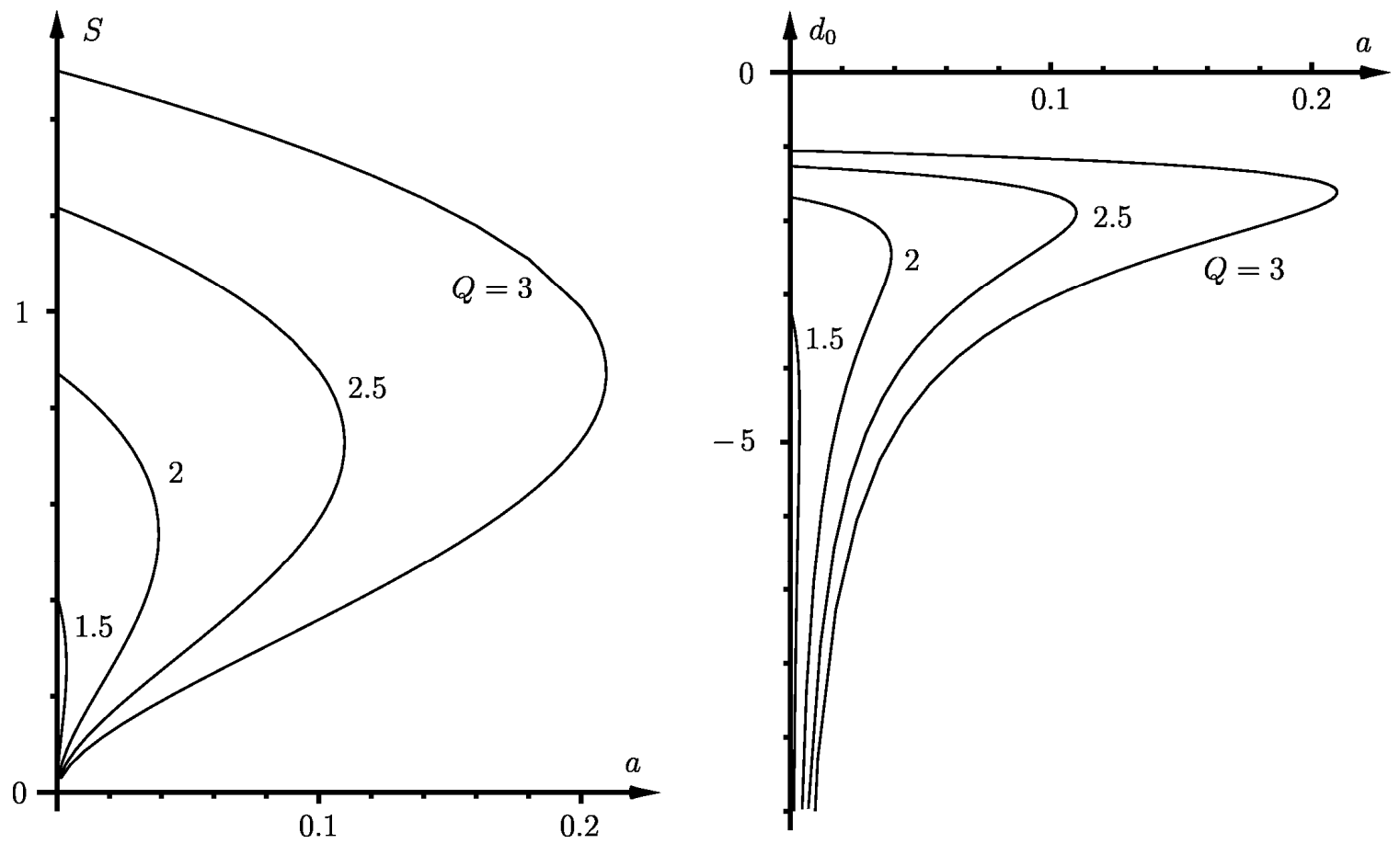

Figure 4: Dependence of the flame speed, $S$ (left figure) and the evaporation front location, $d_{0}$ (right figure) on the heat loss parameter $a$ for selected values of the heat of reaction $Q ; L e_{Z}=1, L=0.2, T_{d}=0.2, \delta=1$, fuel lean flame . 


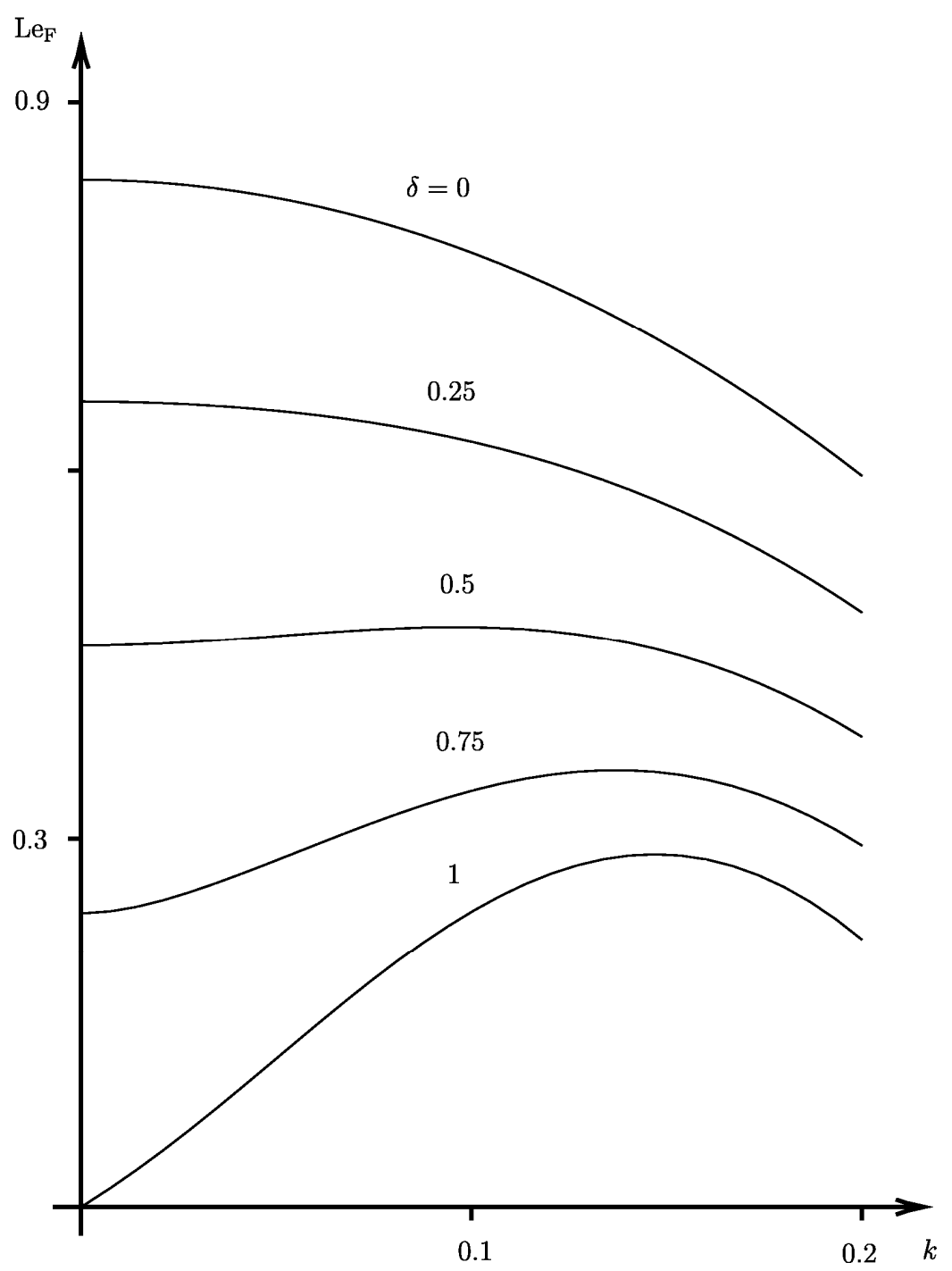

Figure 5: Neutral cellular stability curves for selected values of the initial droplet load for lean spray flames; $a=0, Q=1.2, L e_{Z}=1, L=0.1, T_{d}=0.2$. 


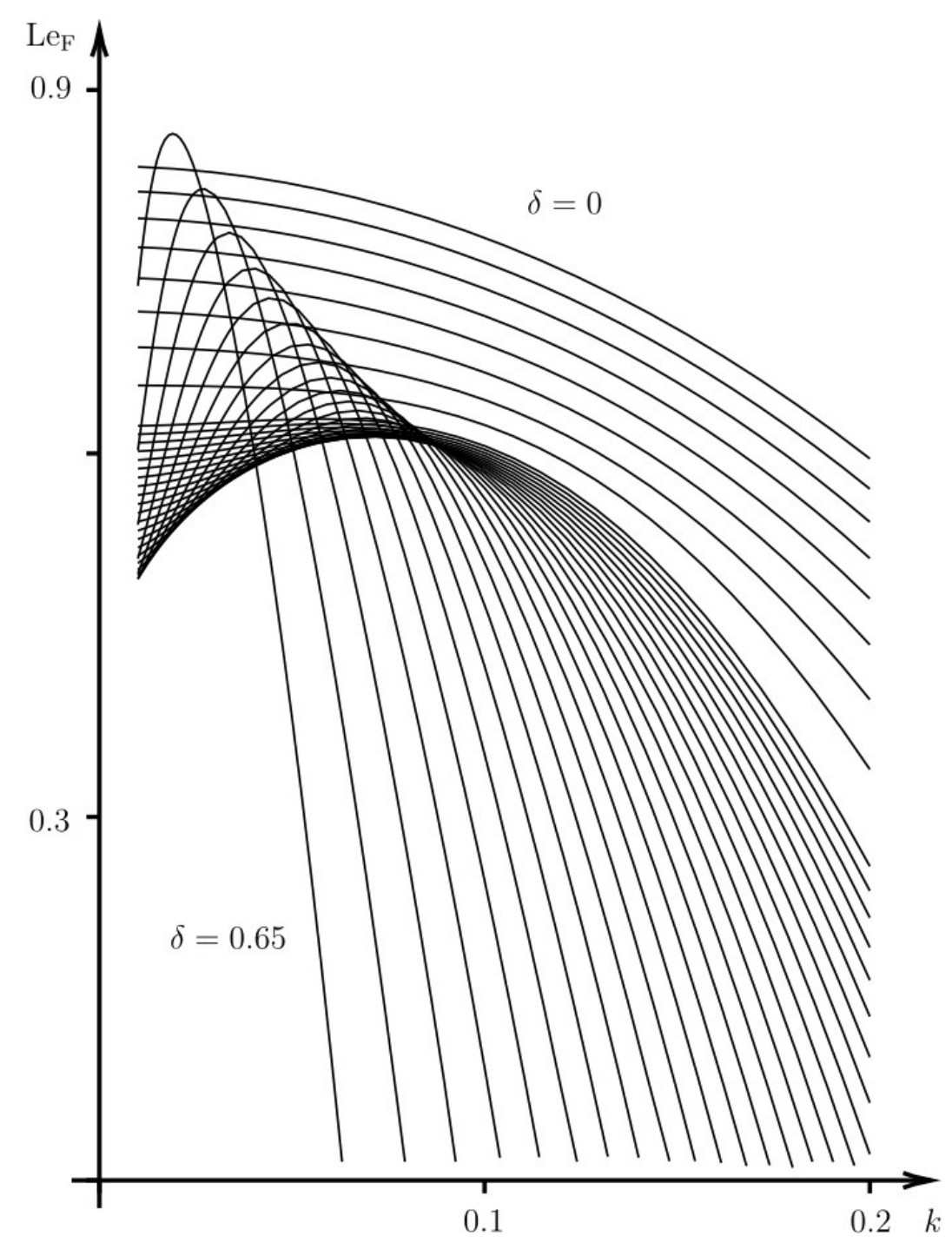

Figure 6: Neutral cellular stability curves for selected values of the initial droplet load for lean spray flames; $a=0, Q=1.2, L e_{Z}=1, L=0.3, T_{d}=0.2$, curves correspond to values of $\delta=0-0.5$ in steps of 0.1 then $\delta=0.54,0.58,0.6,0.62,0.64,0.65$ 

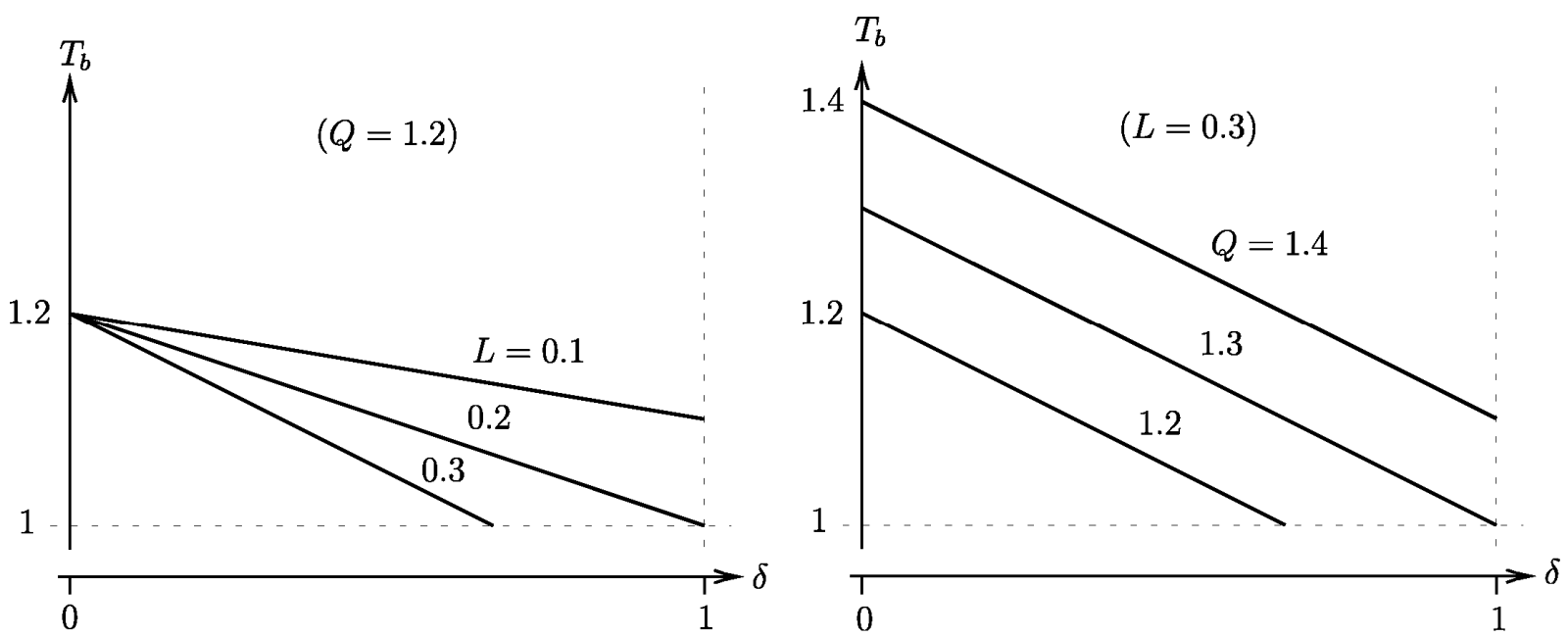

Figure 7: Dependence of flame temperature on liquid droplet load for various values the latent heat of vaporization (left hand figure) and the heat of reaction $Q$ (right hand figure). 


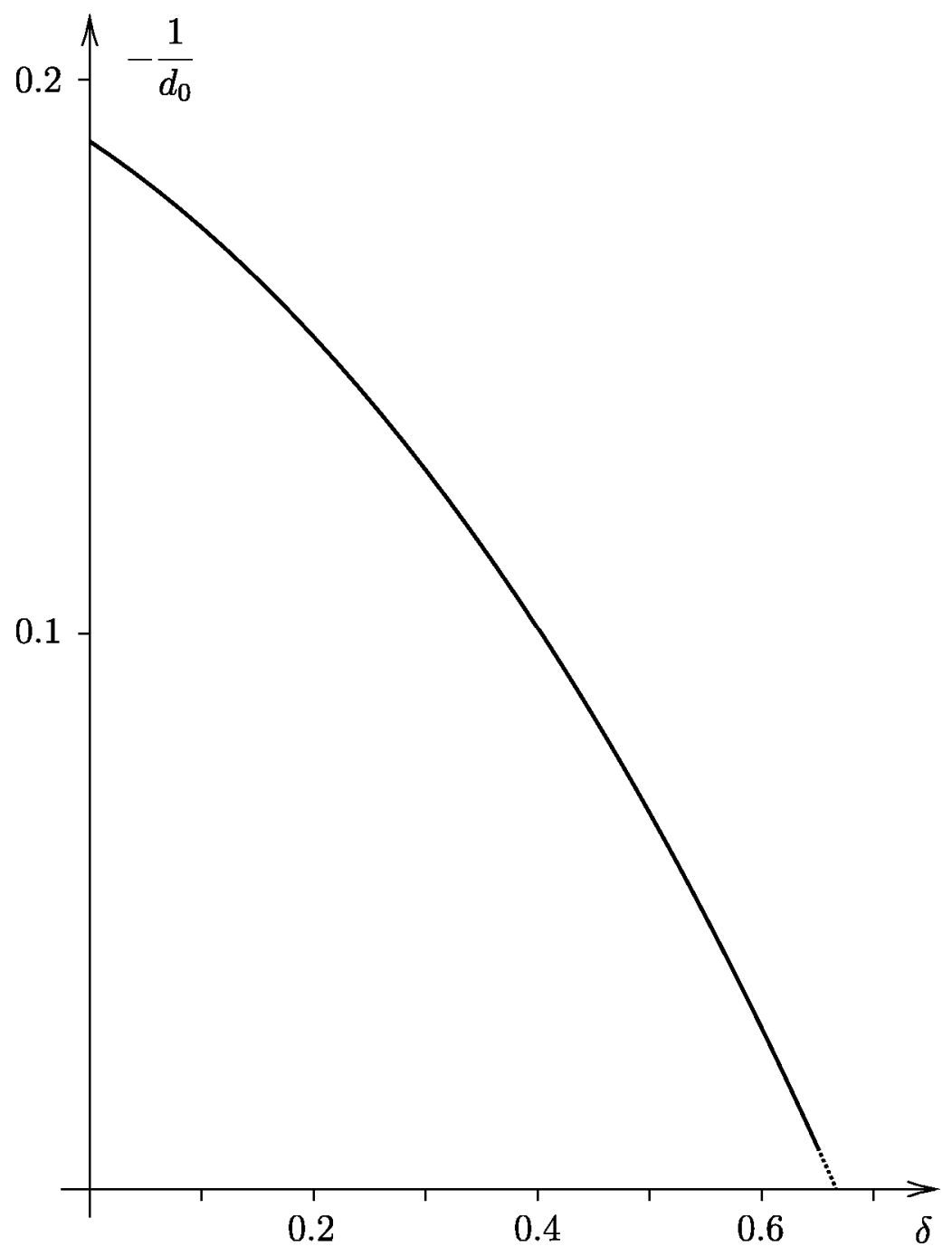

Figure 8: Reciprocal of the evaporation front location as a function of liquid droplet load; data as in Fig. 6. 


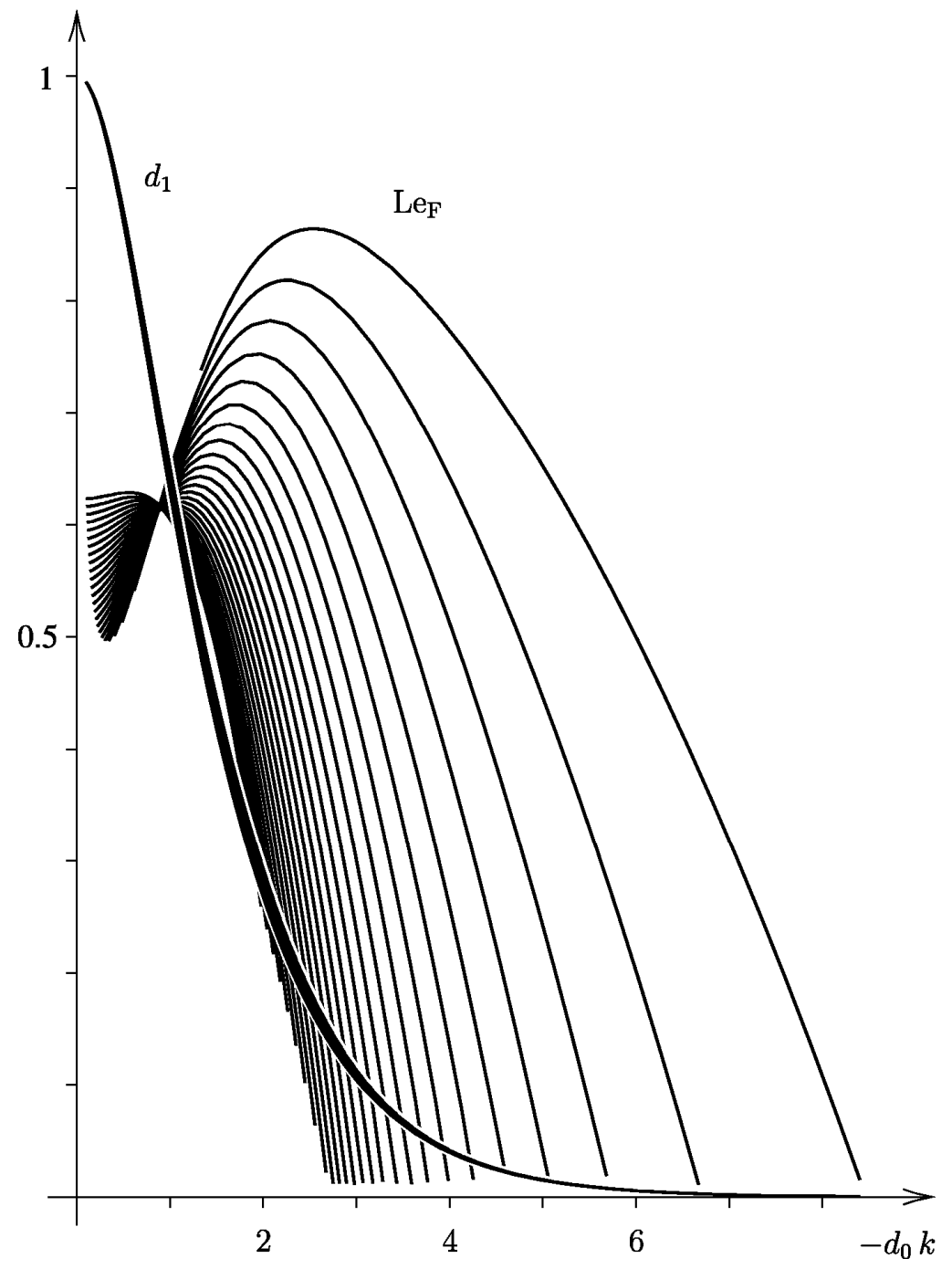

Figure 9: $d_{1}$ and $L e_{F}$ versus $\left(-d_{0} k\right)$ for data of Fig. $6 . \delta$ changes from 0.4 to 0.65 (in steps of 0.01), other data as in Figure 6. Note that as $\delta$ increases the curves of $L e_{F}$ move to the right whereas the curves for $d_{1}$ change very little. 


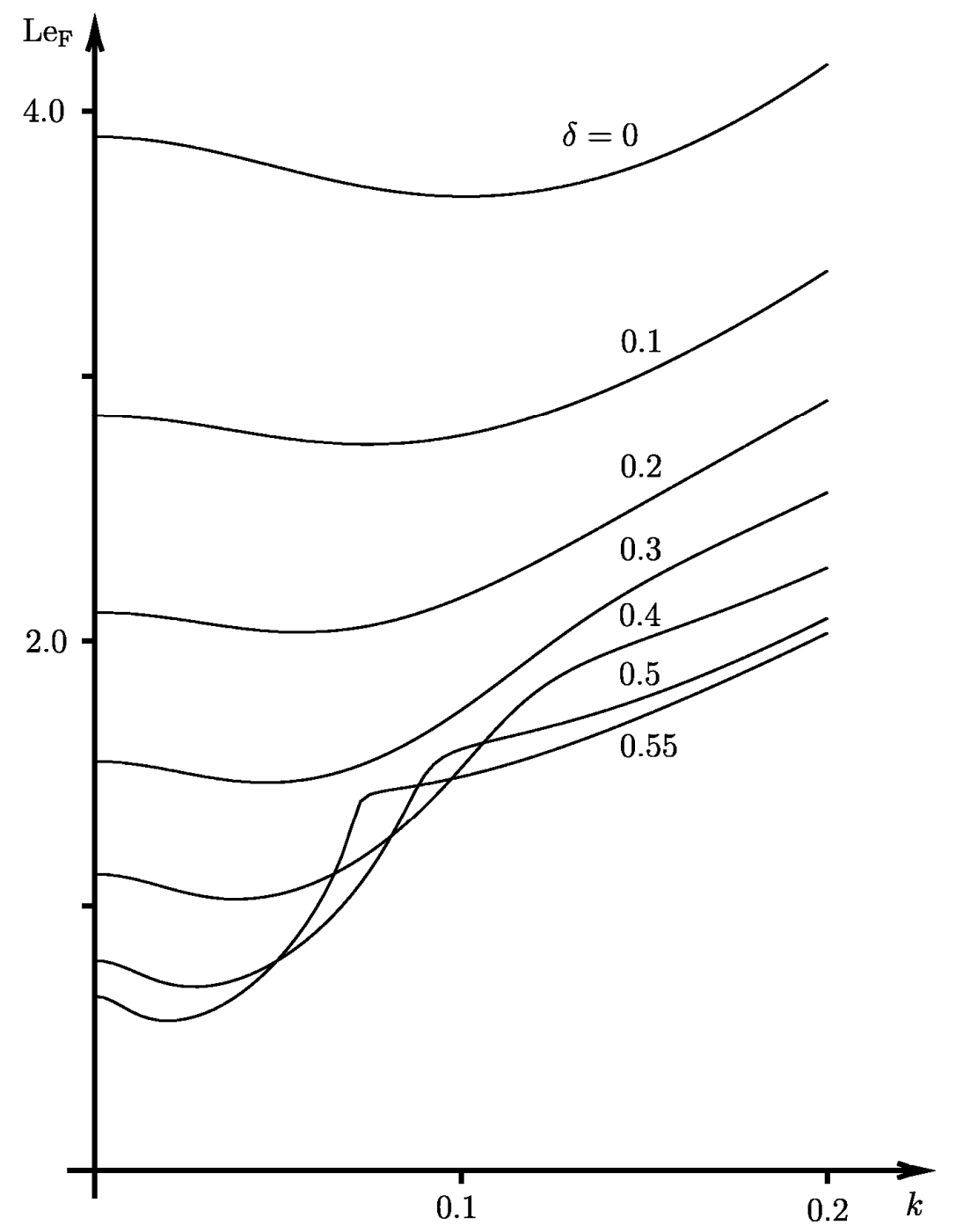

Figure 10: Neutral oscillatory stability curves for selected values of the initial droplet load for fuel lean flames; $a=0, Q=1.2, L e_{Z}=1, L=0.1, T_{d}=0.2$. 

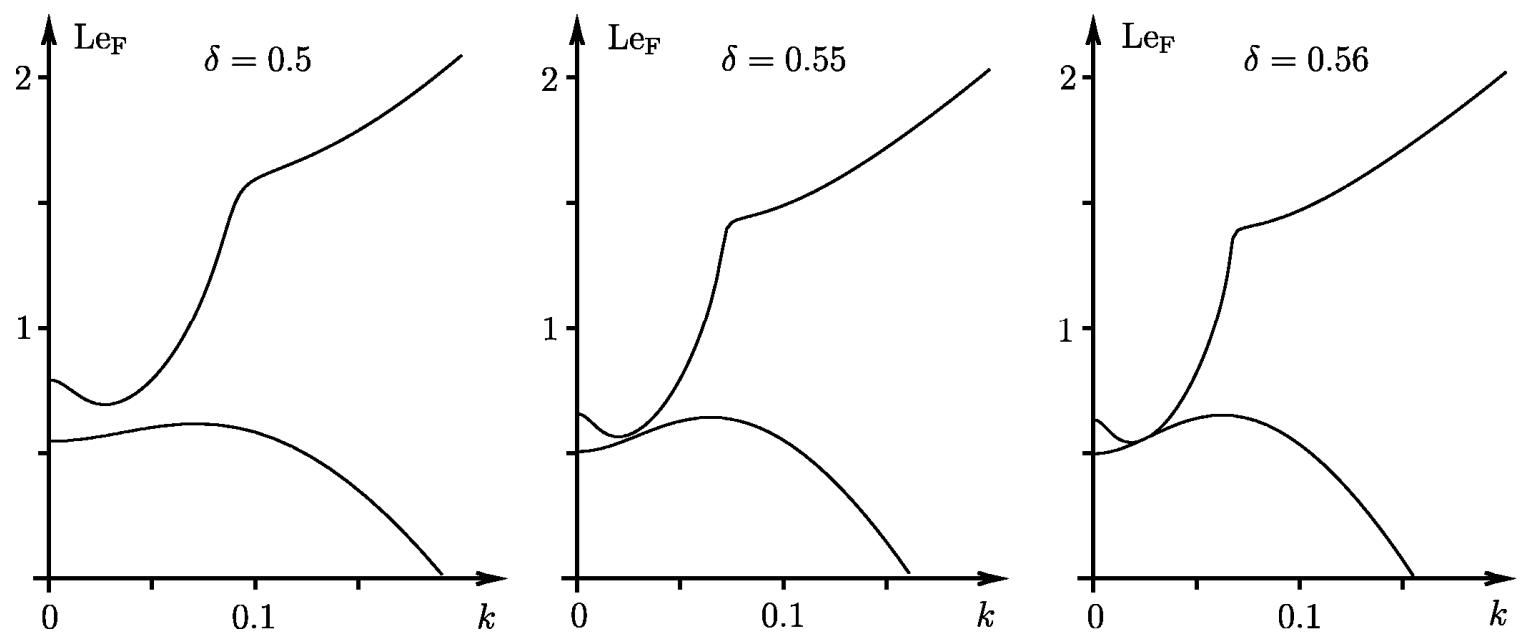

Figure 11: Neutral cellular and oscillatory stability curves for selected values of the initial droplet load for fuel lean spray flames; $a=0, Q=1.2, L e_{Z}=1, L=0.1, T_{d}=0.2$ 


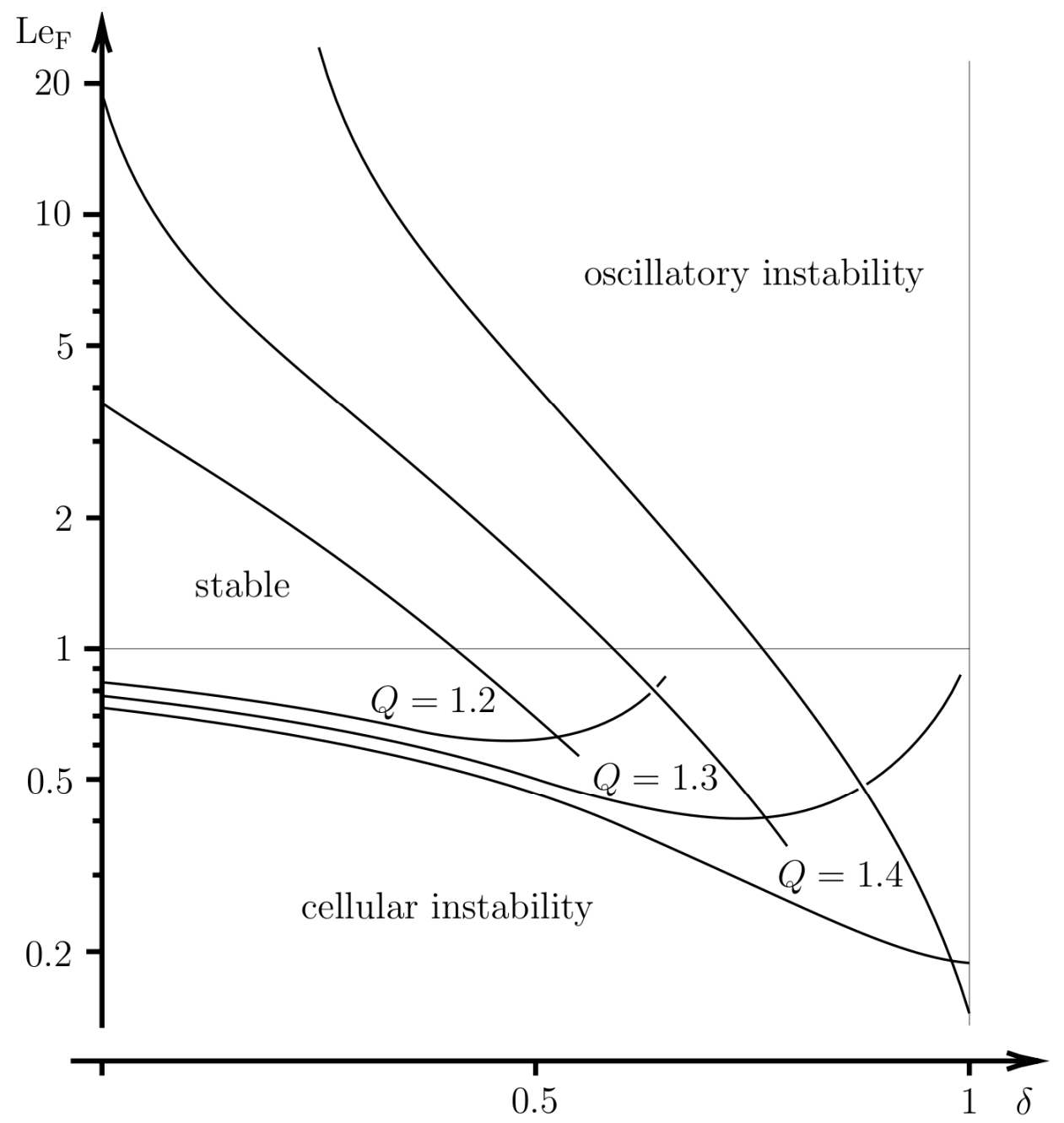

Figure 12: Neutral cellular and oscillatory stability curves for fuel lean spray flames in the $L e_{F}-\delta$ plane for selected values of the heat of the chain breaking reaction $Q$; $a=0, L e_{Z}=1, L=0.3, T_{d}=0.2$. 


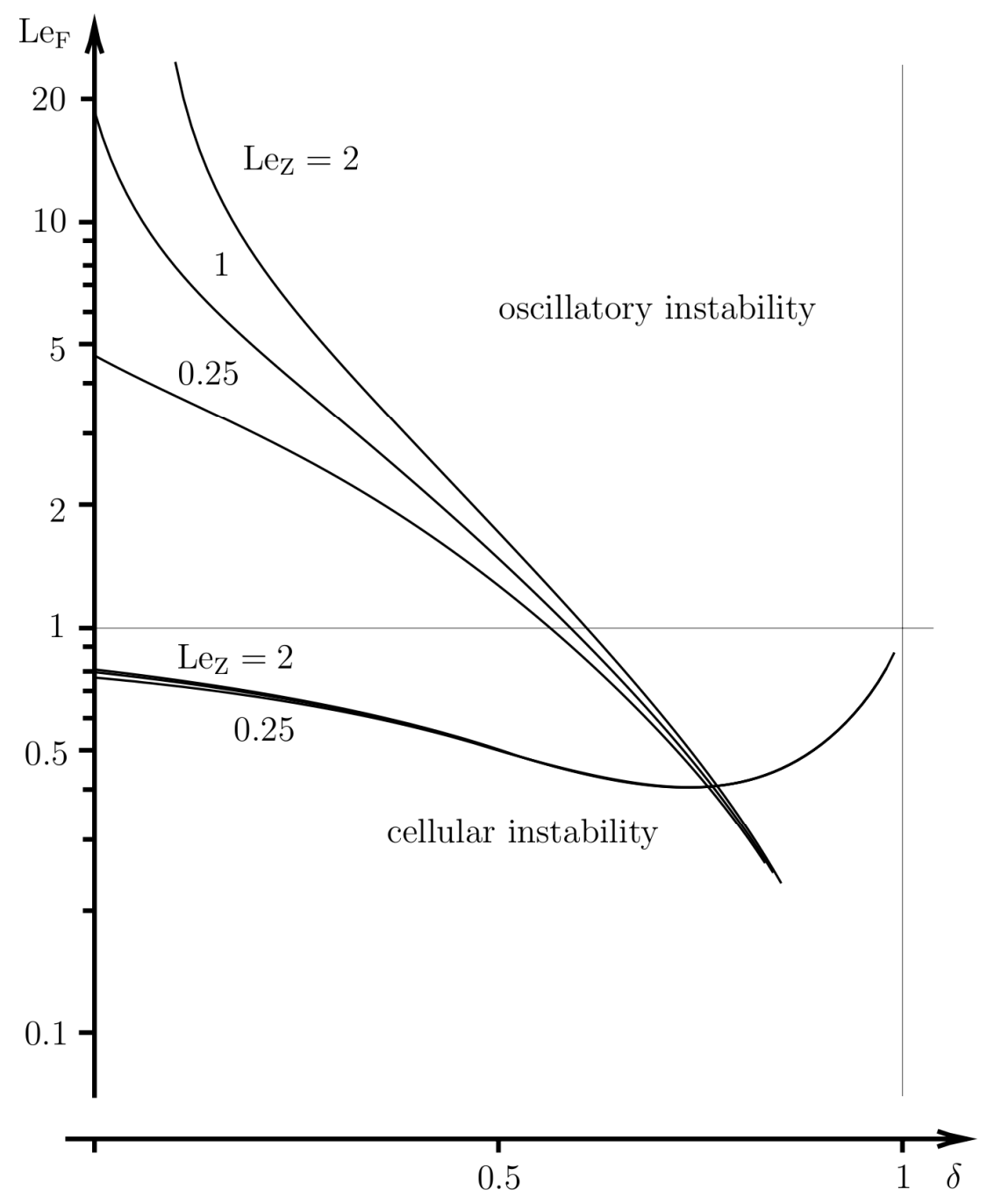

Figure 13: Neutral cellular and oscillatory stability curves for fuel lean spray flames in the $L e_{F}-\delta$ plane for selected values of $L e_{Z} ; a=0, Q=1.3, L=0.3, T_{d}=0.2$. 


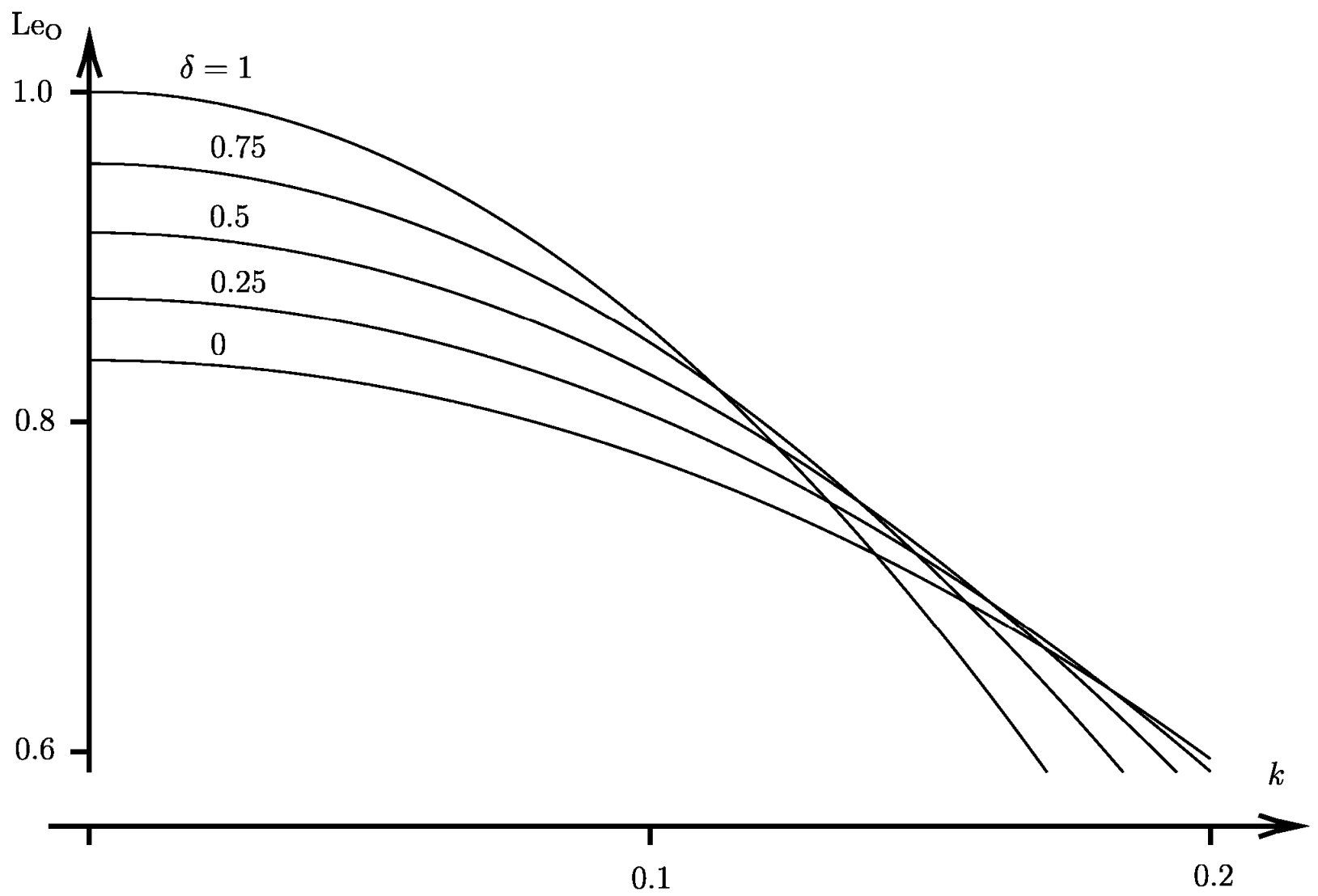

Figure 14: Neutral cellular stability curves for selected values of the initial droplet load for fuel rich spray flames; $a=0, Q=1.2, L e_{Z}=1, L=0.1, T_{d}=0.2$. 


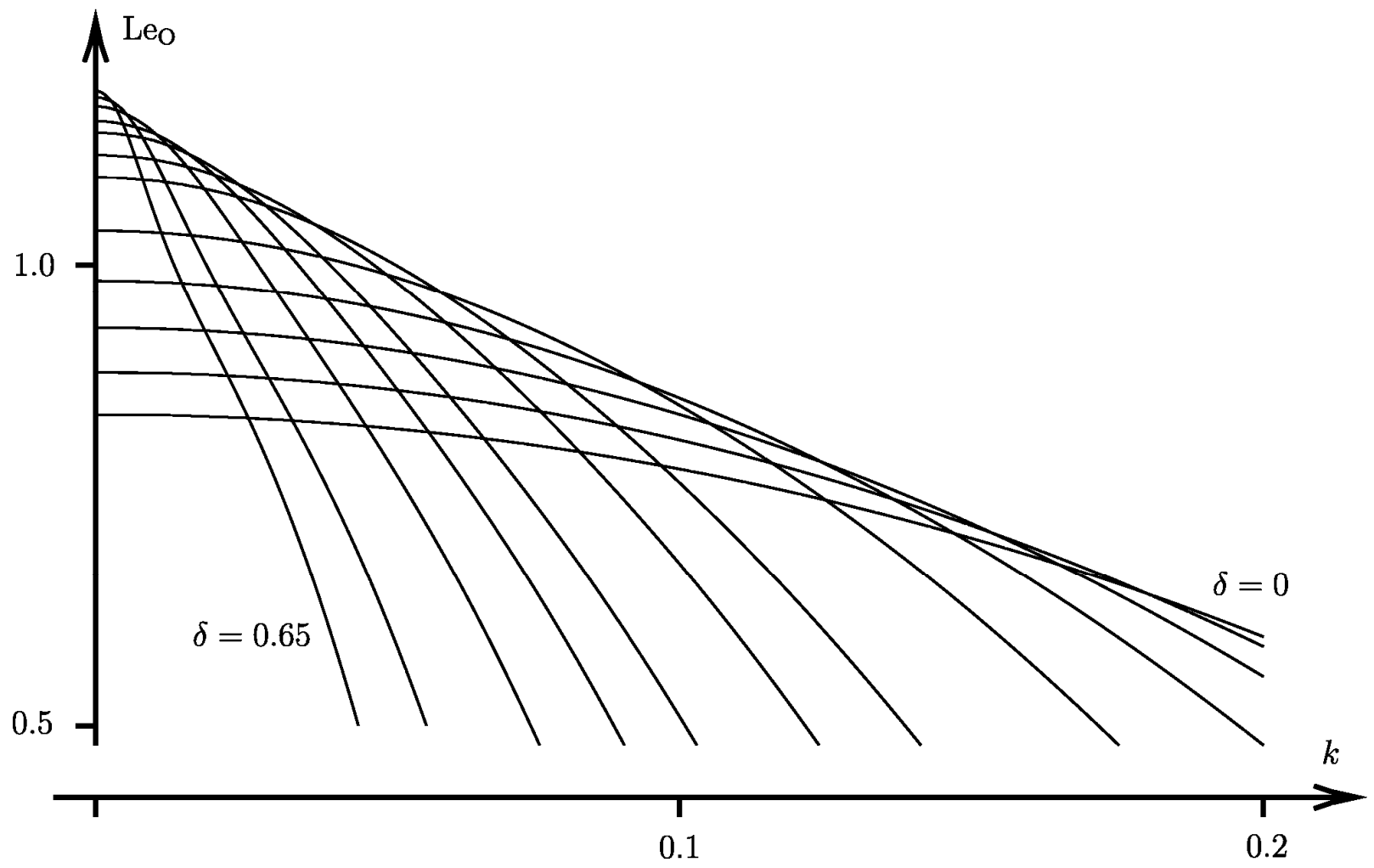

Figure 15: Neutral cellular stability curves for selected values of the initial droplet load for fuel rich spray flames; $a=0, Q=1.2, L e_{Z}=1, L=0.3, T_{d}=0.2$. 


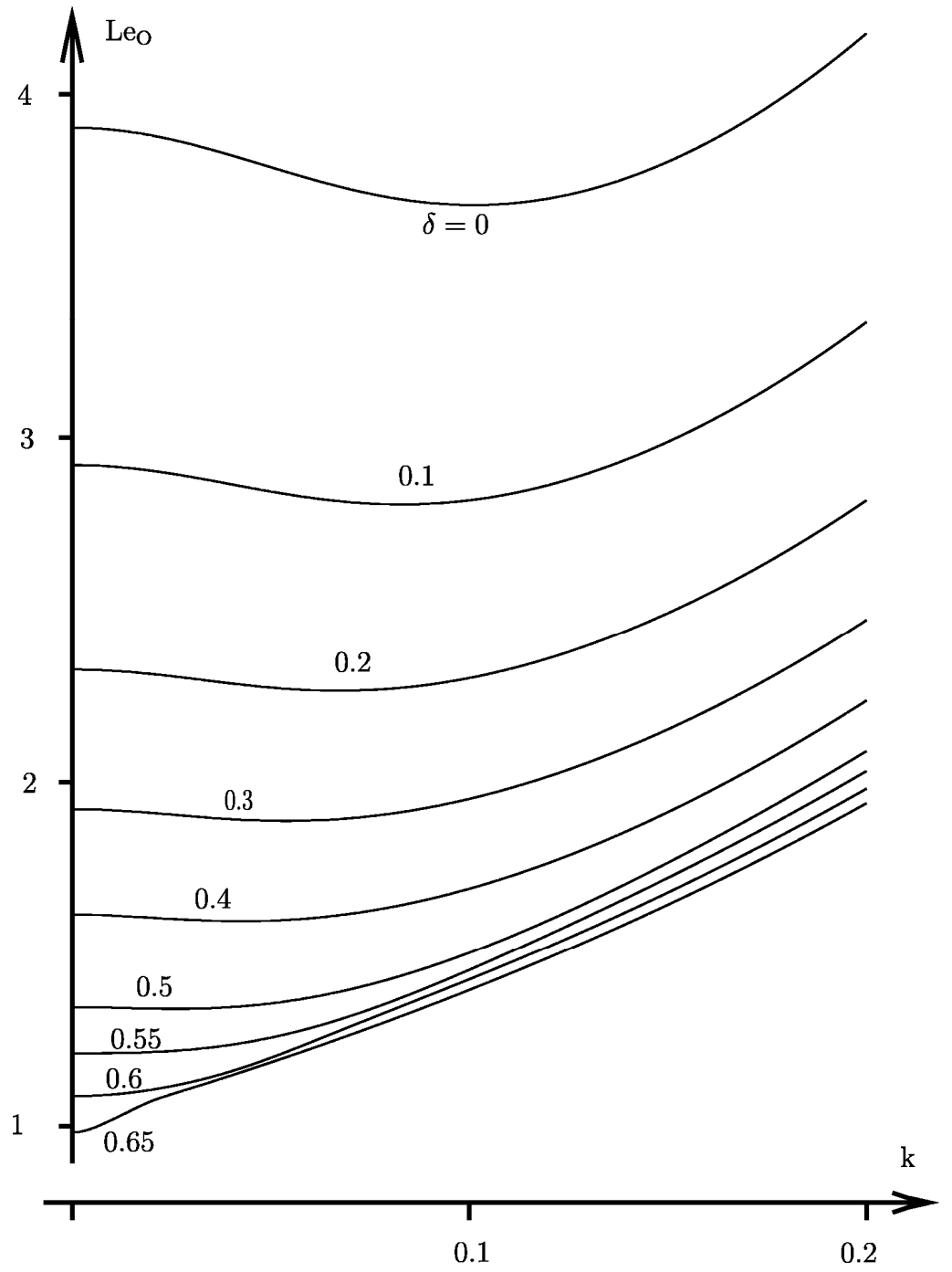

Figure 16: Neutral oscillatory stability curves for selected values of the initial droplet load for fuel rich flames; $a=0, Q=1.2, L e_{Z}=1, L=0.3, T_{d}=0.2$. 


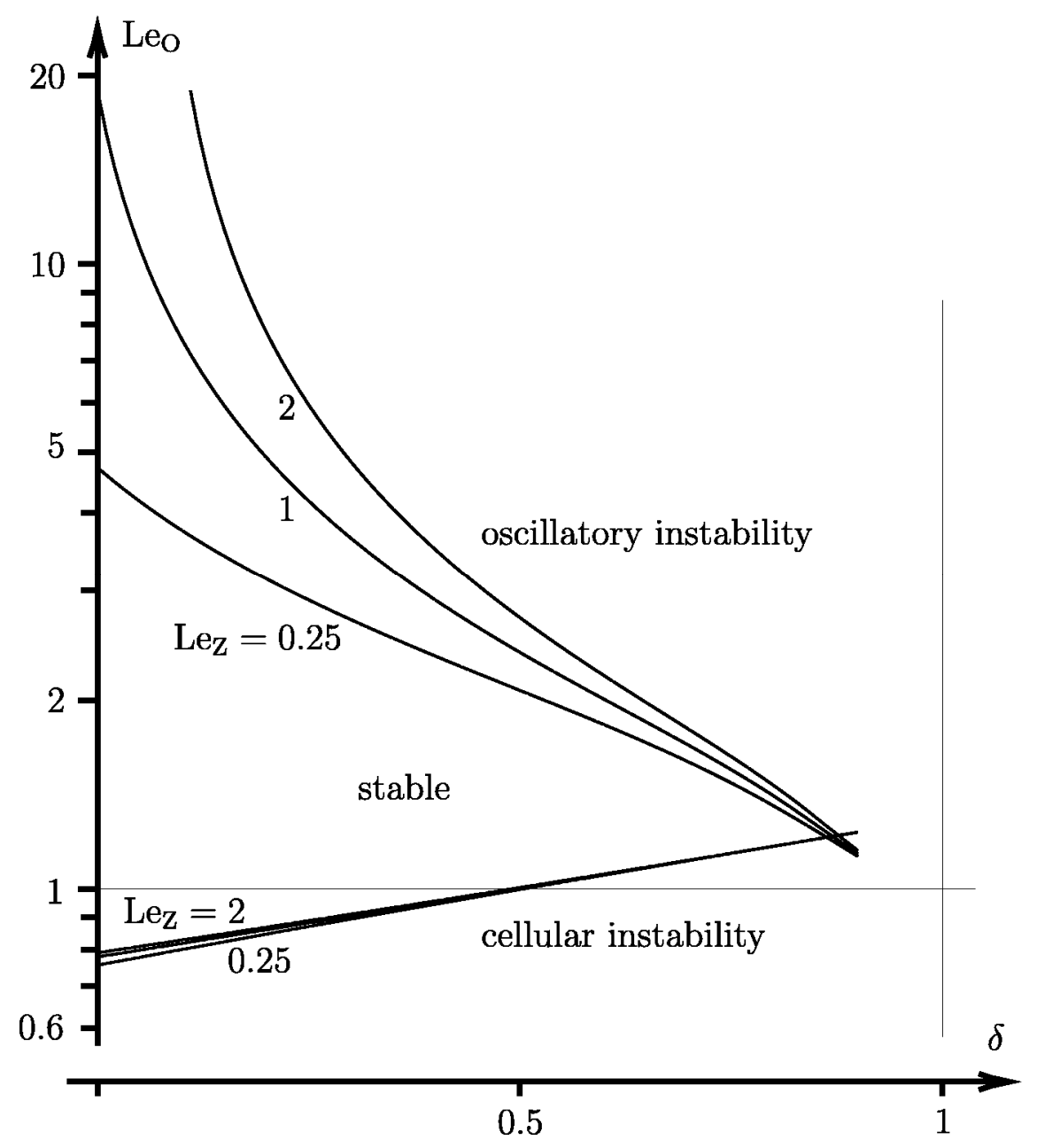

Figure 17: Neutral cellular and oscillatory stability curves for fuel rich spray flames in the $L e_{F}-\delta$ plane for selected values of $L e_{Z} ; a=0, Q=1.3, L=0.3, T_{d}=0.2$. 


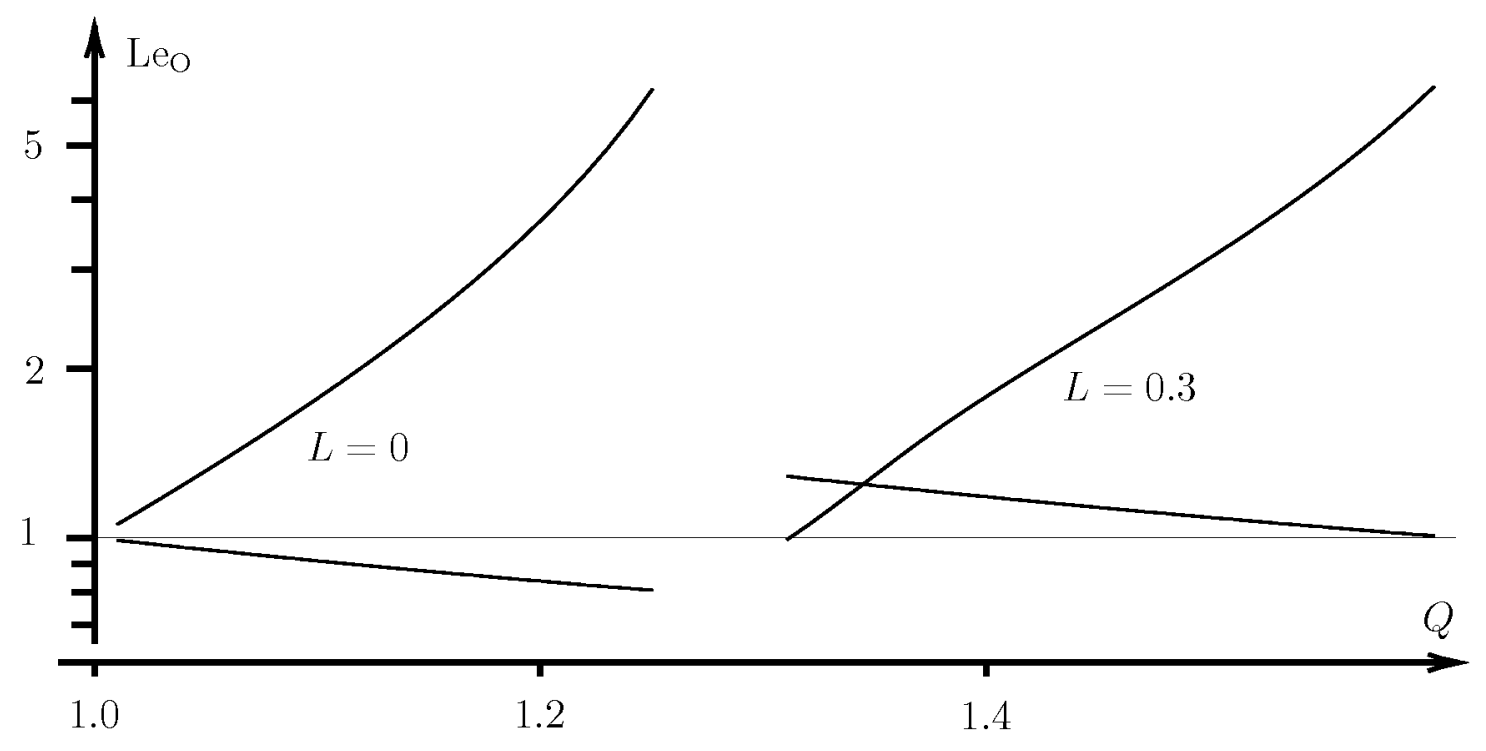

Figure 18: Dependence of $L e_{O}$ on $Q$ at the cellular and oscillatory boundaries of fuel rich spray flames; $\delta=1, T_{d}=0.2, L e_{Z}=1$. 